\begin{tabular}{|c|l|}
\hline Title & Long-time behavior of an electron interacting with a quantized radiation field \\
\hline Author(s) & A rai, A sao \\
\hline Citation & Journal of Mathematical Physics, 32(8), 2224 2242 \\
\hline https:/doi.org/10.1063/1.529197 \\
\hline Issue Date & 1991-08 \\
\hline Doc URL & http://hdl.handle.net/2115/13674 \\
\hline Rights & Copyright $\odot$ 1991 A merican Institute of Physics \\
\hline Type & article \\
\hline File Information & jmp32-81.pdf \\
\hline
\end{tabular}

Instructions for use 


\title{
Long-time behavior of an electron interacting with a quantized radiation field
}

\author{
Asao Arai \\ Department of Mathematics, Hokkaido University, Sapporo 060, Japan
}

(Received 7 August 1990; accepted for publication 19 March 1991)

\begin{abstract}
The long-time behavior of an electron coupled to a quantized radiation field is discussed in the ground state and in equilibrium states at finite temperatures. The electron is not confined in an external potential. The model used is a $d$-dimensional extension of a standard model (the Pauli-Fierz model) in nonrelativistic quantum electrodynamics: The electron moves in $\mathbb{R}^{d}$ $(d \geqslant 2)$ and the radiation field is over $\mathbb{R}^{d}$. Further, the energy function $\omega$ of one free photon is taken to be a general one. In defining the interaction part of the Hamiltonian of the model, an ultraviolet cutoff is introduced for photon momenta with a cutoff function $\hat{\rho}$ and the dipole approximation is used. It is proved that at each finite temperature $T>0$, the mean-square displacement of the electron behaves like $\left(k_{\mathrm{B}} T d / m\right) t^{2}$ as time $t$ tends to infinity, where $k_{\mathrm{B}}$ is the Boltzmann constant and $m>0$ is a renormalized mass of the electron which should be identified with the observed mass of the electron. The long-time asymptotics of the mean square displacement of the electron in the ground state is different from that at the finite temperatures; it depends on the space dimension $d$ and on the infrared behavior of $\omega$ and $\hat{\rho}$.
\end{abstract}

\section{INTRODUCTION}

In this paper we consider an electron coupled to a quantized radiation field. The main purpose is to investigate the long-time behavior of the electron in the ground state and in equilibrium states at finite temperatures in the case where the electron is "free," i.e., not confined in an external potential. For a mathematical generality, we use a model that is a $d$-dimensional extension of a standard model (the PauliFierz model) in nonrelativistic quantum electrodynamics (e.g., Refs. 1-3): The electron moves in $\mathbb{R}^{d}$ and the radiation field is over $\mathbb{R}^{d}(d \geqslant 2)$. Further, we take the energy function $\omega$ of one free photon to be general. This setting has an advantage also in physical considerations, because it may give us a chance to characterize the physical case: $d=3, \omega(\mathbf{k})=|\mathbf{k}|$, $\mathbf{k} \in \mathbb{R}^{d}$. In defining the interaction between the electron and the radiation field, which is taken to be minimal with $\mathbf{A}^{2}$ term retained, an ultraviolet cutoff is introduced for photon momenta with a cutoff function $\hat{\rho}$ and the dipole approximation is used.

Generally speaking, the long-time behavior of a quantum particle interacting with a (random) environment is connected with its transport properties. An interesting question is then whether the particle diffuses or not. A convenient quantity to classify the long-time behavior of the particle, giving also a criterion for the diffusion of the particle, is the mean-square displacement of the particle in a state (e.g., Ref. 4 and references therein). From this point of view, we analyze the long-time behavior of the mean-square displacement of the electron in the ground state and in equilibrium states at finite temperatures. Thus our first task is to establish the existence of the ground state and equilibrium states of the quantum system under consideration. However, this problem is not so trivial. In fact, in the present case, neither the ground state nor equilibrium states may exist, because the electron is not confined in an external potential and the electron and photons move in the continuum $\mathbb{R}^{d}$. Taking this possibility into account, we first consider the situation where the electron is confined in a quadratic potential with a coupling constant $\epsilon>0$, i.e., we treat a model of a harmonically bound electron coupled to a quantized radiation field. Concerning this model, we can show that the ground state and an equilibrium (KMS) state at each finite temperature exist. Then, by taking the "no-binding limit" $\epsilon \rightarrow 0$ of these states in terms of correlation functions, we try to define the ground state and equilibrium states of the quantum system of the free electron coupled to the quantized radiation field. We find that the no-binding limit does not exist for the "usual" correlation functions of the electron position operator. This fact may be interpreted as an indication of nonexistence of the ground state and equilibrium states in the case where the electron is free. We can prove, however, that for the correlation functions of the position displacement operator of the electron, the no-binding limit does exist. In this way we can define a mean-square displacement $\left\langle\Delta q(t)^{2}\right\rangle_{M}(\beta)$ of the free electron coupled to the quantized radiation field in an equilibrium state at each finite temperature $\beta{ }^{\cdots 1}$ and in the ground state $(\beta=\infty)$, where $\mathbf{q}(t)$ is the position operator of the electron at time $t, \Delta \mathbf{q}(t)=\mathbf{q}(t)-\mathbf{q}$, and $M \geqslant 0$ is a parameter denoting the "photon mass." We show that for all $\beta \in(0, \infty)$ and $M \geqslant 0$,

$$
\left\langle\Delta \mathbf{q}(t)^{2}\right\rangle_{M}(\beta) \sim(d / m \beta) t^{2}
$$

as $t \rightarrow \infty$, where $m>0$ is a renormalized mass of the electron that should be identified with the observed mass of the electron (Theorems 4.6 and 4.9). Formula (1.1) means that at finite temperatures, the electron diffuses with an infinite diffusion constant. On the other hand, we see that the long-time aymptotics of the mean-square displacement of the electron in the ground state is different from (1.1); it depends on the space dimension $d$ and on the infrared behavior of $\omega$ and the cutoff function $\hat{\rho}$ (Theorem 3.5).

One might argue that the use of the dipole approximation may be physically questionable in the case under consideration, i.e., in the situation where the electron moves far 
away from the origin, because the dipole approximation may be valid only if the electron stays close near the origin. Unfortunately, we do not have any answer to this question at present. We leave this problem for future studies. The present model should be regarded as a mathematical one, which may still be meaningful as a model of a quantum (Brownian) particle interacting with a heat bath (e.g., cf. Ref. 5). The present work should be considered a preliminary one toward analysis of the long-timc behavior of the Pauli-Ficrz model without the dipole approximation. ${ }^{6}$

The outline of the present paper is as follows. Following the strategy stated above, we first consider in Sec. II a model of a harmonically bound electron coupled to a quantized radiation field. We introduce an ultraviolet cutoff $\hat{\rho}$ for photon momenta in the interaction between the electron and the radiation field, so that the Hamiltonian of the model can be realized as a self-adjoint operator in the Fock space of the electron and the radiation field. The model is a $d$-dimensional extension of the model discussed in Ref. 7 in that (i) the electron and photons move in $\mathbb{R}^{d}(d \geqslant 2)$; (ii) the energy $\omega(\mathbf{k})$ of a free photon with momentum $\mathbf{k} \in \mathbb{R}^{d}$ is taken to be general, including the standard case $\omega(\mathbf{k})=\sqrt{|\mathbf{k}|^{2}+M^{2}}$. In Ref. 7 , the physical case $[d=3, \omega(\mathbf{k})=|\mathbf{k}|]$ was discussed to analyze the mathematical structure of the "Lamb shift" and the spontaneous emission of light (cf. also Refs. 8-13). We show that the present model makes a difference between the massless case $M=0$ and the massive case $M>0$. The method to analyze the present model is quite similar to that in Ref. 7. Thus we shall state almost all of the results without proof. The main results in this section include: (i) the identification of the spectra of the total Hamiltonian $H$; (ii) the existence of the ground state of $H$; (iii) derivation of explicit formulas of the vacuum expectation values (VEVs) for Heisenberg operators.

In Sec. III we consider the no-binding limit $\epsilon \rightarrow 0$ of the model in the ground state. We show that for the VEVs of the electron position operator, the no-binding limit does not exist, but, for the VEVs of $\Delta \mathbf{q}(t)$, it does if $M>0$; we derive an explicit formula for $\left\langle\Delta q(t)^{2}\right\rangle_{M}$, the mean-square displacement of the electron in the ground state. The mean-square displacement of the electron in the case $M=0$ is defined as the limit of $\left\langle\Delta \mathbf{q}(t)^{2}\right\rangle_{M}$ as $M \rightarrow 0$. We prove the following facts: (i) if $M>0$, then $\left\langle\Delta q(t)^{2}\right\rangle_{M}$ is bounded in $t$ and hence the electron does not diffuse; (ii) if $M=0$, then the longtime asymptotics of $\left\langle\Delta \mathbf{q}(t)^{2}\right\rangle_{M}$ depends on $d$ and on the infrared behavior of $\omega$ and $\hat{\rho}$. Moreover, we show that the VEVs of the electron velocity $\mathbf{v}(t)=d \mathbf{q}(t) / d t$ have the nobinding limits.

In Sec. IV we discuss the case where the quantum system of the electron and the radiation field is in a finite temperature state. We first construct an equilibrium (KMS) state $\langle\cdot\rangle_{M, \epsilon}(\beta)$ of the model discussed in Sec. II and derive explicit formulas for correlation functions of the clectron. As in the case of the ground state, we show that the no-binding limit does not exist for the "usual" correlation functions of the electron position operator, but, for the correlation functions of $\Delta \mathbf{q}(t)$, it does exist. In particular, we derive an explicit formula for the no-binding limit of the mean-square displacement:

$$
\left\langle\Delta \mathbf{q}(t)^{2}\right\rangle_{M}(\beta)=\lim _{\epsilon \rightarrow 0}\left\langle\Delta \mathbf{q}(t)^{2}\right\rangle_{M, \epsilon}(\beta)
$$

Then we analyze the long-time behavior of $\left\langle\Delta \mathbf{q}(t)^{2}\right\rangle_{M}(\beta)$ and prove (1.1). The correlation functions of the electron velocity are also discussed. We show that they have the nobinding limits and, in the no-binding limit, the correlation between $\mathbf{v}(t)$ and $\mathbf{v}(0)$ persists as $|t| \rightarrow \infty$.

In Appendix A we give an estimate of a one-dimensional integral, which is used in the text to prove the nonexistence of the no-binding limit of the VEVs or the correlation functions of $\mathbf{q}(t)$. In Appendix B we establish an elementary limit theorem for a one-dimensional integral, which is applied in the text to derive the long-time asymptotic formulas of the mean-square displacement of the electron.

\section{A HARMONICALLY BOUND ELECTRON COUPLED TO A QUANTIZED RADIATION FIELD}

Models of a harmonically bound electron coupled to a quantized radiation field have been discussed from various points of view (e.g., Refs. 7-13, see also Refs. 14-19 for their scalar field versions). In this section we reconsider one of such models in a mathematically rigorous way and summarize some fundamental properties of it. The model here is a $d$ dimensional extension of the model discussed in Ref. 7. The functional analytic method used in Ref. 7 still works without any significant modification, giving results similar to those obtained there. Thus most of the results in this section will be given without detailed proofs.

\section{A. Definition of the model}

The Hilbert space of state vectors for the harmonically bound electron is $L^{2}\left(\mathbb{R}^{d}\right)$. We use the system of units where the Planck constant divided by $2 \pi$ and the speed of light are 1 , respectively. We denote by $\mathbf{q}=\left(q_{1}, \ldots, q_{d}\right) \in \mathbb{R}^{d}$ the position operator of the electron. The electron momentum operator is given by

$$
\mathbf{p}=\left(-i \frac{\partial}{\partial q_{1}}, \ldots,-i \frac{\partial}{\partial q_{d}}\right)=-i \nabla,
$$

where the differential operators $\partial / \partial q_{j}, j=1, \ldots, d$, are taken in the generalized sense.

We use the Coulomb gauge in quantizing the free classical radiation field in the Fock representation, so that the Hilbert space of state vectors for the free quantized radiation field is the Boson Fock space

$$
\mathscr{F}_{\mathrm{EM}}=\underset{n=0}{\oplus} \otimes_{s}^{n} W
$$

over the (one-photon) Hilbert space

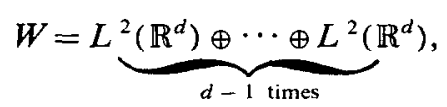

where $\otimes{ }_{s}^{n} W$ denotes the $n$-fold symmetric tensor product of $W$ with convention $\otimes_{s}^{n=0} W=\mathbb{C}$. Let $\mathbf{a}(\mathbf{f}), \mathbf{f} \in W$, be the usual annihilation operator in $\mathscr{F}_{\mathrm{EM}}$ (antilinear in $\mathbf{f}$ ). For $r=1, \ldots, d-1$, and $f \in L^{2}\left(\mathbb{R}^{d}\right)$, we define $\mathbf{f}_{r} \in W$ by $\mathbf{f}_{r}=(0, \ldots, f, \ldots, 0)$ (the $r$ th component is equal to $f$ and the other components are zero). The mapping: $f \rightarrow \mathbf{a}\left(\mathbf{f}_{r}\right)$ defines an operator-valued distribution on $\mathbb{R}^{d}$. We denote the distri- 
bution kernel by $a_{r}(\mathbf{k}), \mathbf{k} \in \mathbb{R}^{d}$. The operator-valued distributions $a_{r}(\mathbf{k})$ and $a_{r}(\mathbf{k})^{*}$ satisfy the standard canonical commutation relations.

Let $\omega_{1}(t)$ be a continuously differentiable, strictly monotone increasing function on $(0, \infty)$ such that $\omega_{1}(t) \rightarrow \infty$ as $t \rightarrow \infty$ and

$$
\omega_{1}(0) \equiv \inf _{t>0} \omega_{1}(t)=0
$$

and define the rotation invariant function

$$
\omega(\mathbf{k})=\omega_{1}\left(\sqrt{|\mathbf{k}|^{2}+\boldsymbol{M}^{2}}\right)
$$

on $\mathbb{R}^{d}$, where $M \geqslant 0$ is a parameter. We take $\omega(\mathbf{k})$ to be the energy of a free photon with momentum $\mathbf{k}$. The standard physical choice for $\omega(\mathbf{k})$ is given by the case $\omega_{1}(t)=t$, so that $\omega(\mathbf{k})=\sqrt{|\mathbf{k}|^{2}+M^{2}}$. In this case, $M$ means the "photon mass" (usually $M=0$ ). We set

$$
\omega_{0}=\inf _{\mathbf{k} \in \mathbf{R}^{d}} \omega(\mathbf{k})=\omega_{\mathrm{I}}(\boldsymbol{M}) .
$$

The free Hamiltonian of the quantized radiation field is defined by

$$
H_{F}=\int d \mathbf{k} \omega(\mathbf{k}) a_{r}(\mathbf{k}) * a_{r}(\mathbf{k}),
$$

where summation over repeated indices with respect to $r=1, \ldots, d-1$, is understood.

The Hilbert space $\mathscr{F}$ of state vectors for the interacting system of the electron and the radiation field is taken to be the tensor product of $L^{2}\left(\mathbb{R}^{d}\right)$ and $\mathscr{F}_{\mathrm{FM}}$ :

$$
\mathscr{F}=L^{2}\left(\mathbb{R}^{d}\right) \otimes \mathscr{F}_{\mathrm{EM}} .
$$

We denote by $\Omega_{0}$ the Fock vacuum in $\mathscr{F}_{\mathrm{EM}}$ and define $\mathscr{F}_{\text {EM }, 0}$ to be the subspace of $\mathscr{F}_{\mathrm{EM}}$ spanned by vectors of the form

$$
\mathbf{a}\left(\mathbf{f}_{1}\right)^{*} \cdots \mathbf{a}\left(\mathbf{f}_{n}\right) * \Omega_{0}, \quad \Omega_{0}, \quad \mathbf{f}_{j} \in W, j=1, \ldots, n, n \geqslant 1 .
$$

We set

$$
\mathscr{F}_{0}=C_{0}^{\infty}\left(\mathbb{R}^{d}\right) \hat{\otimes} \mathscr{F}_{\mathrm{EM}, 0},
$$

where $\hat{\otimes}$ denotes algebraic tensor product. The subspace $\mathscr{F}_{0}$ is dense in $\mathscr{F}$.

A densely defined closed linear operator $A$ (resp. $B$ ) in $L^{2}\left(\mathbb{R}^{d}\right)$ (resp. $\mathscr{F}_{\mathrm{EM}}$ ) can be extended to an operator $\mathscr{F}$ as $A \otimes I$ (resp. $I \otimes B$ ), where $I$ denotes identity. For notational simplicity, we shall denote the extensions by the same symbols.

Let $\mathbf{e}^{(r)}(\mathbf{k})$ be an $\mathbb{R}^{d}$-valued measurable function on $\mathbb{R}^{d}$ such that

$$
\begin{aligned}
& \mathbf{k} \cdot \mathbf{e}^{(r)}(\mathbf{k})=0, \quad \mathbf{e}^{(r)}(\mathbf{k}) \cdot \mathrm{e}^{(s)}(\mathbf{k})=\delta_{r s}, \\
& \text { a.e. } \mathbf{k} \in \mathbb{R}^{d}, \quad r, s=1, \ldots, d-1 .
\end{aligned}
$$

The vectors $\mathrm{e}^{(r)}(\mathrm{k}), r=1, \ldots, d-1$, serve as polarization vectors of a photon with momentum $k$. The time-zero radiation field $\mathbf{A}(\mathbf{x})$ and its conjugate $\pi(\mathbf{x})$ are defined, respectively, as an operator-valued distribution of the following form:

$\mathbf{A}(\mathbf{x})=\int d \mathbf{k} \frac{1}{\sqrt{2 \omega(\mathbf{k})}} \mathbf{e}^{(r)}(\mathbf{k})\left(a_{r}(\mathbf{k})^{*} e^{-i \mathbf{k} \cdot \mathbf{x}}+a_{r}(\mathbf{k}) e^{i \mathbf{k}-\mathbf{x}}\right)$,

$$
\begin{aligned}
\pi(\mathbf{x})= & i \int d \mathbf{k} \sqrt{\frac{\omega(\mathbf{k})}{2}} \mathrm{e}^{(r)}(\mathbf{k}) \\
& \times\left(a_{r}(\mathbf{k})^{*} e^{-i \mathbf{k} \cdot \mathbf{x}}-a_{r}(\mathbf{k}) e^{i \mathbf{k} \cdot \mathbf{x}}\right), \quad \mathbf{x} \in \mathbb{R}^{d} .
\end{aligned}
$$

Let $\rho$ be a real-valued measurable function on $\mathbb{R}^{d}$ such that its Fourier transform

$$
\hat{\rho}(\mathbf{k})=\frac{1}{(2 \pi)^{d / 2}} \int d \mathbf{x} \rho(\mathbf{x}) e^{-i \mathbf{k} \cdot \mathbf{x}}
$$

exists with $\omega^{-1 / 2} \hat{\rho} \in L^{2}\left(\mathbb{R}^{d}\right)$. We shall use $\hat{\rho}$ as a cutoff function for large photon momenta. The time-zero radiation field with cutoff $\rho$ is then defined by

$$
\begin{aligned}
\mathbf{A}(\mathbf{x} ; \rho) \equiv & \int \rho(x-y) \mathbf{A}(y) d \mathbf{y} \\
\equiv & \int d \mathbf{k} \frac{1}{\sqrt{2 \omega(\mathbf{k})}} \mathbf{e}^{(r)}(\mathbf{k}) \\
& \times\left\{\hat{\rho}(\mathbf{k})^{*} a_{r}(\mathbf{k})^{*} e^{-\mathbf{k} \cdot \mathbf{x}}+\hat{\rho}(\mathbf{k}) a_{r}(\mathbf{k}) e^{i \mathbf{k} \times \mathbf{x}}\right\}
\end{aligned}
$$

which is essentially self-adjoint on $\mathscr{F}_{0}$. We take the interaction between the electron and the quantized radiation field to be minimal and use the dipole approximation. Thus the total Hamiltonian of the model we are going to study is defined by

$$
H=\left(1 / 2 m_{0}\right)(\mathbf{p}-e \mathbf{A}(0 ; \rho))^{2}+H_{F}+\frac{1}{2} \epsilon \mathbf{q}^{2},
$$

where $m_{0}>0$ (resp. $\epsilon>0, e \in \mathbb{R} \backslash\{0\}$ ) is a parameter denoting the bare mass of the electron (resp. the spring constant of the harmonically bound electron, the elementary charge) (cf. Refs. 1-3). The free Hamiltonian of the model is obtained by putting $e=0$ in $H$ :

$$
H_{0}=\left(1 / 2 m_{0}\right) \mathbf{p}^{2}+(\epsilon / 2) \mathbf{q}^{2}+H_{F}
$$

which is self-adjoint and non-negative with domain $D\left(H_{0}\right)=D\left(\mathbf{p}^{2}\right) \cap D\left(\mathbf{q}^{2}\right) \cap D\left(H_{F}\right)$.

Proposition 2.1: Suppose that $\hat{\rho} / \omega, \omega^{1 / 2} \hat{\rho} \in L^{2}\left(\mathbb{R}^{d}\right)$. Then $H$ is self-adjoint, non-negative with domain $D(H)=D\left(H_{0}\right)$ and essentially self-adjoint on every core of $H_{0}$.

Proof: Similar to the proof of Theorem A.2 of Ref. 7 (cf. also Theorem 3.1 in Ref. 14). The idea of the proof is to apply the Glimm-Jaffe-Nelson commutator theorem [e.g., Ref. 20 (Sec. 19.4) and Ref. 21 (Sec. X.5)] with the "test operator" $H_{0}$, where the following estimates are used:

$$
\begin{gathered}
\left\|a_{r}(f)^{\#} \Psi\right\| \leqslant\left\|\omega^{-1 / 2} f\right\|_{L^{2}}\left\|H_{F}^{1 / 2} \Psi\right\|+\|f\|_{L^{2}}\|\Psi\|, \\
\Psi \in D\left(H_{F}^{1 / 2}\right), r=1, \ldots, d-1 .
\end{gathered}
$$

$\left[a_{r}(f)^{\#}\right.$ denotes either $a_{r}(f)$ or $\left.a_{r}(f)^{*}.\right]$

Remark: We can also show that $D\left(H^{1 / 2}\right)=D\left(H_{0}^{1 / 2}\right)$.

\section{B. Heisenberg operators}

By Proposition 2.1 the total Hamiltonian $H$ generates the unitary group $\left\{e^{i t H} ; t \in \mathbb{R}\right\}$, which gives the dynamics of the quantum system under consideration. The time evolution of the dynamical variables $\{\mathbf{q}, \mathbf{p}, \mathbf{A}(\mathbf{x}), \pi(\mathbf{x})\}$ are defined by the Heisenberg operators

$$
\begin{aligned}
& \mathbf{q}(t)=e^{i t H} \mathbf{q} e^{-i t H}, \\
& \mathbf{p}(t)=e^{i t t h} \mathbf{p} e^{-i t H},
\end{aligned}
$$




$$
\begin{aligned}
& \mathbf{A}(t, \mathbf{x})=e^{i t H} \mathbf{A}(\mathbf{x}) e^{-i t H}, \\
& \pi(t, \mathbf{x})=e^{i t H} \pi(\mathbf{x}) e^{-i t H}, \quad t \in \mathbb{R} .
\end{aligned}
$$

We also define

$$
\mathbf{A}(t, \mathbf{x} ; \rho)==e^{i t H} \mathbf{A}(\mathbf{x} ; \rho) e^{-i t H} .
$$

In the same way as in Ref. 7, one can show that these operators are defined on $D\left(H_{0}^{1 / 2}\right)$ and satisfy the following equations of motion on $\mathscr{F}_{0} \cap D\left(H_{0}^{2}\right)$ (in the sense of operatorvalued distribution with respect to variable $\mathbf{x}$ ):

$$
\begin{aligned}
& \left(m_{0} \frac{d^{2}}{d t^{2}}+\epsilon\right) \mathbf{q}(t)=-e \frac{\partial}{\partial t} \int d \mathbf{x} \rho(\mathbf{x}) \mathbf{A}(t, \mathbf{x}), \\
& \left(\frac{\partial^{2}}{\partial t^{2}}+\omega(-i \nabla)^{2}\right) A_{\mu}(t, \mathbf{x})=e \rho_{\mu v}(\mathbf{x}) \frac{d}{d t} q_{v}(t), \\
& \quad \mu=1, \ldots, d . \\
& m_{0} \frac{d}{d t} \mathbf{q}(t)=\mathbf{p}(t)-e \mathbf{A}(t, 0 ; \rho), \\
& \frac{\partial}{\partial t} \mathbf{A}(t, \mathbf{x})=\pi(t, x) .
\end{aligned}
$$

Here the time derivatives are taken in the strong sense, $\omega(-i \nabla)$ is the pseudodifferential operator defined by

$$
\omega(-\imath \nabla) f(\mathbf{x})=\frac{1}{(2 \pi)^{d / 2}} \int \omega(\mathbf{k}) \hat{f}(\mathbf{k}) e^{i \mathbf{k} \cdot \mathbf{x}} d \mathbf{k}
$$

and

$$
\rho_{\mu v}(\mathbf{x})=\frac{1}{(2 \pi)^{d / 2}} \int d \mathbf{k} \hat{\rho}(\mathbf{k}) d_{\mu v}(\mathbf{k}) e^{i \mathbf{k} \cdot \mathbf{x}},
$$

with

$$
d_{\mu v}(\mathbf{k}) \equiv e_{\mu}^{(r)}(\mathbf{k}) e_{v}^{(r)}(\mathbf{k})=\delta_{\mu v}-k_{\mu} k_{v} /|\mathbf{k}|^{2} .
$$

Summation over repeated indices with respect to Greek letters is also understood.

The equations of motion (2.21)-(2.24) are exactly solvable, which allows us to represent explicitly the Heisenberg operators given by $(2.16)-(2.19)$. To do that, however, we need some technical considerations. We first define a class of the cutoff function $\rho$.

Definition 2.2: Let $\rho$ be a real-valued measurable function on $\mathbb{R}^{d}$. We say that $\rho$ is in $\mathscr{K}\left(\mathbb{R}^{d}\right)$ if the Fourier transform $\hat{\rho}$ is a measurable function on $\mathbb{R}^{d}$ and depends only on $|\mathbf{k}|$ with

$$
\hat{\rho}(\mathbf{k})>0, \quad \mathbf{k} \in \mathbb{R}^{d} \backslash\{0\},
$$

and

$$
\int d \mathbf{k} \frac{\hat{\rho}(\mathbf{k})^{2}}{\omega(\mathbf{k})^{2}-\omega_{0}^{2}}<\infty, \quad \int d \mathbf{k} \omega(\mathbf{k}) \hat{\rho}(\mathbf{k})^{2}<\infty,
$$

where $\omega_{0}$ is defined by (2.5).

We shall write also as $\hat{\rho}(\mathbf{k})=\hat{\rho}(|\mathbf{k}|)$. We remark that $\rho \in \mathscr{K}\left(\mathbb{R}^{d}\right)$ is allowed to tend to zero as $\mathbf{k} \rightarrow 0$. The infrared behavior of $\hat{\rho}(\mathbf{k})$ affects the long-time asymptotics of correlation functions of the model under consideration (cf. Ref. 22, see also Secs. III and IV).

If $\rho \in \mathscr{K}\left(\mathbb{R}^{d}\right)$, then $\rho$ satisfies the assumption of Proposition 2.1. For $p \in \mathscr{K}\left(\mathbb{R}^{d}\right)$, we define a function $D(z)$ of complex variable $z$ by with

$$
D(z)=\epsilon-z m(z),
$$

$$
m(z)=m_{0}+\frac{(d-1) e^{2}}{d} \int d \mathbf{k} \frac{\hat{\rho}(\mathbf{k})^{2}}{\omega(\mathbf{k})^{2}-z} .
$$

The functions $m(z)$ and $D(z)$ are analytic in the complex cut plane

$$
\mathbb{C}_{\omega_{0}} \equiv \mathbb{C} \backslash\left[\omega_{0}^{2},+\infty\right) .
$$

We set

$$
\epsilon_{0}=\omega_{0}^{2} m\left(\omega_{0}^{2}\right)
$$

and

$$
m \equiv m(0)=m_{0}+\frac{(d-1) e^{2}}{d} \int d \mathbf{k} \frac{\hat{\rho}(\mathbf{k})^{2}}{\omega(\mathbf{k})^{2}} .
$$

Lemma 2.3: (i) Let $\omega_{0}=0$. Then $D(z)$ has no zeros in $\mathbb{T}_{0}$.

(ii) Let $\omega_{0}>0$ and $\epsilon<\epsilon_{0}$. Then $D(z)$ has a unique zero $\lambda(\epsilon)^{2} \in\left(0, \omega_{0}^{2}\right)$ of order 1 [we take $\lambda(\epsilon)>0$ ]. Further we have

$$
\lambda(\epsilon)^{2}<\epsilon / m,
$$

for all $\epsilon \in\left(0, \epsilon_{0}\right)$ and

$$
\lambda(\epsilon)^{2}=\frac{\epsilon}{m}-\frac{(d-1) e^{2}\left\|\omega^{-2} \hat{\rho}\right\|_{L^{2}}^{2}}{m d}\left(\frac{\epsilon}{m}\right)^{2}+O\left(\epsilon^{3}\right)
$$

as $\epsilon \rightarrow 0$.

(iii) Let $\omega_{0}>0$ and $\epsilon>\epsilon_{0}$. Then $D(z)$ has no zeros in $\mathbb{C}_{\omega_{0}}$

(iv) Let $\omega_{0}>0$ and $\epsilon=\epsilon_{0}$. Then $D(z)$ has no zeros in $\mathbb{C}_{\omega_{0}}$, but, $D\left(\omega_{0}^{2}\right)=0$.

Proof: An elementary exercise.

Remark: The constant $m$ given by (2.30) is a renormalized mass of the clectron. A scattering-theoretical analysis in the case $\epsilon=0$ shows that it should be identified with the observed mass of the electron. ${ }^{3}$

Let $\eta:\left(\omega_{0}, \infty\right) \rightarrow(0, \infty)$ be the inverse function of $\omega_{1}\left(\sqrt{t^{2}+M^{2}}\right)$ :

$$
\eta(t)=\sqrt{\omega_{1}^{-1}(t)^{2}-M^{2}},
$$

where $\omega_{1}^{-1}$ is the inverse function of $\omega_{1}$, and define

$K_{M}(x)=\left\{\begin{array}{l}(2 \sqrt{x})^{-1}|\hat{\rho}(\eta(\sqrt{x}))|^{2} \eta(\sqrt{x})^{d-1} \eta^{\prime}(\sqrt{x}), \\ x>\omega_{0}^{2}, \\ 0, \quad x \leqslant \omega_{0}^{2} .\end{array}\right.$

In what follows, we assume the following.

Assumption $K$ (Lipshitz condition):

$\left|K_{M}(x+h)-K_{M}(x)\right| \leqslant C|h|^{\alpha}$

uniformly in $x \in \mathbb{R}$, as $h \rightarrow 0$, with some constants $C>0$ and $0<\alpha<1$ independent of $M$ sufficiently small.

This assumption is not empty. For example, if

$$
d>\max \{2,2 p\}, \quad \omega_{1}(t)=t^{p} \quad(p>0),
$$

and $\hat{\rho}(k)=$ const $k^{n} \exp \left(-c k^{2}\right) \quad(c>0, n \geqslant 0$ :integer $)$, then Assumption $K$ is satisfied. A general sufficient condition for Assumption $K$ to hold is discussed in Appendix A of Ref. 22. 
Assumption $K$ and the fact $K_{M} \in L^{1}(\mathbb{R})$ ensure that $K_{M}$ is bounded with

$$
\lim _{x \rightarrow \infty} K_{M}(x)=0
$$

and its Hilbert transform

$$
I_{M}(x)=\frac{1}{\pi} \text { p.v. } \int_{-\infty}^{\infty} \frac{K_{M}(y)}{x-y} d y
$$

exists for all $x \in \mathbb{R}$, satisfying a Lipshitz condition with the same $\alpha$ as $K_{M}$ [e.g., Ref. 23 (Sec. 5.15)]:

$$
\left|I_{M}(x+h)-I_{M}(x)\right| \leqslant C^{\prime}|h|^{\alpha}
$$

uniformly in $x \in \mathbb{R}$, as $h \rightarrow 0$, with a positive constant $C^{\prime}$ independent of $M$ sufficiently small.

Let $d S(\theta)$ be the canonical surface measure on the $(d-1)$-sphere $S^{d-1}$ and $V_{d}$ be the volume of $S^{d-1}$ :

$$
V_{d}=\int_{S^{d}}, d S(\theta)=\frac{2 \pi^{d / 2}}{\Gamma(d / 2)},
$$

where $\Gamma(z)$ is the gamma function. Set

$$
\gamma_{d}=V_{d}(d-1) e^{2} / d \text {. }
$$

Lemma 2.4: For all $x \geqslant \omega_{0}^{2}$, the limits

$$
m_{ \pm}(x ; M) \equiv \lim _{\delta \rightarrow+0} m(x \pm i \delta)
$$

exist and are given by

$$
m_{ \pm}(x ; M)=m_{0}-\pi \gamma_{d}\left\{I_{M}(x) \mp i K_{M}(x)\right\} .
$$

Moreover,

$$
\inf _{x>\omega_{0}^{2}}\left|m_{-}(x ; M)\right| \geqslant C,
$$

with a positive constant $C$ independent of $M$ sufficiently small.

Proof: We can write $m(x \pm i \delta)=m_{0}-\gamma_{d}\left\{\left(Q_{\delta} * K_{M}\right)(x) \mp i\left(P_{\delta} * K_{M}\right)(x)\right\}$, where $P_{\delta}$ and $Q_{\delta}$ are the Poisson and the conjugate Poisson kernel, respectively,

$$
P_{\delta}(x)=\delta /\left(x^{2}+\delta^{2}\right), \quad Q_{\delta}(x)=x /\left(x^{2}+\delta^{2}\right),
$$

and $*$ denotes convolution. Assumption $K$ allows us to apply a general theory of Fourier analysis (e.g., Ref. 23) to obtain (2.38).

To prove (2.39), we note that

$$
\left|m_{-}(x ; M)\right|^{2}=\left(m_{0}-\pi \gamma_{d} I_{M}(x)\right)^{2}+\gamma_{d}^{2} \pi^{2} K_{M}(x)^{2} .
$$

Since $I_{M}(x)$ is continuous in $x$, we have

$$
\lim _{x \rightarrow \omega_{0}^{2}}\left\{m_{0}-\pi \gamma_{d} I_{M}(x)\right\}=m\left(\omega_{0}^{2}\right)>0 .
$$

By (2.35) this convergence is uniform in $\omega_{0}<\alpha_{0}$, where $\alpha_{0}>0$ is a sufficiently small constant. For $0<\omega_{0}<\alpha_{0}$, we have

$$
m\left(\omega_{0}^{2}\right) \geqslant m_{0}+\frac{(d-1) e^{2}}{d} \int \frac{\hat{\rho}(\mathbf{k})^{2}}{\omega_{1}\left(\sqrt{\mathbf{k}^{2}+M_{0}^{2}}\right)} \equiv A_{0},
$$

where

$$
\omega_{1}\left(M_{0}\right)=\alpha_{0} .
$$

Hence, for every $\epsilon \in\left(0, A_{0}\right)$, there exists a positive constant $\delta$ independent of $\omega_{0}\left(<\alpha_{0}\right)$ such that

$$
\inf _{\omega_{0}^{2}<x<\omega_{0}^{2}+\delta}\left\{m_{0}-\pi \gamma_{d} I_{M}(x)\right\}^{2} \geqslant\left(A_{0}-\epsilon\right)^{2},
$$

which implies that for $0 \leqslant \omega_{0}<\alpha_{0}$

$$
\inf _{\omega_{0}^{2}<x<\omega_{0}^{2}+\delta}\left|m_{-}(x ; M)\right|^{2} \geqslant\left(A_{0}-\epsilon\right)^{2} .
$$

Since $K_{M}(x)$ is bounded and $K_{M} \in L^{1}(\mathbb{R})$, it follows that $K_{M} \in L^{p}(\mathbb{R})$ for all $p \in[1, \infty)$. Hence, $I_{M} \in L^{p}(\mathbb{R})$ for $p \in(1, \infty)$. Using this fact and $(2.35)$, we have

$$
\sup _{M<M_{0}}\left|I_{M}(x)\right| \rightarrow 0
$$

as $x \rightarrow \infty$ [cf. Ref. 23 (Sec. 5.15)]. Therefore, for every $\epsilon \in\left(0, m_{0}\right)$, there exists a constant $R>0$ independent of $M<M_{0}$ such that for all $x>R$

$$
\inf _{x>R}|m \ldots(x ; M)|^{2}>\left(m_{0}-\epsilon\right)^{2} .
$$

We have

$$
\inf _{\omega_{0}^{2}+\delta \leqslant x \leqslant R} \hat{\rho}(\eta(\sqrt{x})) \geqslant \inf _{\delta_{1} \leqslant k \leqslant \omega_{1} \cdot l^{\prime}(\sqrt{R})} \hat{\rho}(k) \equiv C_{1}>0,
$$

where

$$
\delta_{1}=\inf _{M<M_{0}} \sqrt{\omega_{1}^{-1}\left(\sqrt{\delta+\omega_{0}^{2}}\right)^{2}-M^{2}}>0,
$$

with $M_{0}$ taken to be sufficiently small. Further, using (2.32), we can show that

$$
\inf _{\omega_{0}^{2}+\delta_{i x \leqslant R}} \frac{\eta(\sqrt{x})^{d-1} \eta^{\prime}(\sqrt{x})}{2 \sqrt{x}} \geqslant C_{2}\left(\omega_{0}\right)
$$

with a constant $C_{2}\left(\omega_{0}\right)>0$ satisfying

$$
\lim _{\omega_{0} \rightarrow 0} C_{2}\left(\omega_{0}\right)>0
$$

Hence, we have

$$
\inf _{\omega_{0}^{2}+\delta_{* i x \leqslant R}} K_{M}(x)^{2} \geqslant C>0,
$$

with a constant $C$ independent of $\omega_{0}$ sufficiently small. Combining this estimate with $(2.40)$ and (2.41), we obtain (2.39).

Using Lemma 2.4 and (2.26), we see that the limits

$$
D_{ \pm}(x)=\lim _{\delta \rightarrow+10} D(x \pm i \delta)
$$

exist for all $x \geqslant \omega_{0}^{2}$ and are given by

$$
D_{ \pm}(x)=\epsilon-x m_{ \pm}(x ; M) .
$$

In the case $\epsilon \neq \epsilon_{0}$, we have

$$
D_{ \pm}(x) \neq 0, \quad x \geqslant \omega_{0}^{2} .
$$

Hence, we can define

$$
F_{\epsilon}(\mathrm{k})=-i e \frac{\hat{\rho}(\mathrm{k})}{D_{-}\left(\omega(\mathrm{k})^{2}\right)},
$$

for which a remarkable integral formula holds.

Lemma 2.5: Let $f(x)$ be a measurable function on $\left[\omega_{0}, \infty\right)$ such that $\left|f(\omega(\mathbf{k})) \| F_{\epsilon}(\mathbf{k})\right|^{2}$ is integrable on $\mathbb{R}^{d}$ with respect to $d \mathbf{k}$. Then 


$$
\begin{aligned}
& \left(\frac{d-1}{d}\right) \int d \mathbf{k} f(\omega(\mathbf{k}))\left|F_{\epsilon}(\mathbf{k})\right|^{2} \\
& =\frac{2}{\pi} \int_{\omega_{0}}^{\infty} d x \frac{f(x)}{x} \operatorname{Im} D_{+}\left(x^{2}\right)^{-1}
\end{aligned}
$$

Proof: Let $I(f)$ be the left-hand side (lhs) of (2.44). By the change of variable $x=\omega_{1}\left(\sqrt{k^{2}+M^{2}}\right)$, we have

$$
I(f)=\gamma_{d} \int_{\omega_{0}}^{\infty} d x \frac{f(x) \hat{\rho}(\eta(x))^{2} \eta(x)^{d-1} \eta^{\prime}(x)}{\left|D_{-}\left(x^{2}\right)\right|^{2}} .
$$

By using formulas (2.38) and (2.42), we see that

$$
\begin{aligned}
\gamma_{d} & \frac{\hat{\rho}(\eta(x))^{2} \eta(x)^{d-1} \eta^{\prime}(x)}{\left|D_{-}\left(x^{2}\right)\right|^{2}} \\
& =\frac{1}{i \pi x}\left\{\frac{1}{D_{+}\left(x^{2}\right)}-\frac{1}{D_{-}\left(x^{2}\right)}\right\} \\
& =\frac{2}{\pi x} \operatorname{Im} D_{+}\left(x^{2}\right)^{-1} .
\end{aligned}
$$

Hence (2.44) follows.

In the case where $\omega_{0}>0$ and $\epsilon<\epsilon_{0}$, we introduce

$c_{\epsilon}=\theta\left(\omega_{0}\right) \theta\left(\epsilon_{0}-\epsilon\right)\left[\lambda(\epsilon) \sqrt{-D^{\prime}\left(\lambda(\epsilon)^{2}\right)}\right]^{-1}$,

where $\theta(t)$ is the Heaviside function: $\theta(t)=1$ for $t>0$ and $\theta(t)=0$ for $t<0$. lemma.

An important property of $F_{\epsilon}$ is given by the following

Lemma 2.6: Let $\epsilon \neq \epsilon_{0}$ in the case $\omega_{0}>0$. Let $g(z)$ be a meromorphic function on $\mathbb{C} \cup\{\infty\}$ and $\left\{a_{n}\right\}_{n-1}^{N}(N<\infty)$ be its poles with $a_{n} \in \mathbb{C}_{\omega_{0}} \backslash\left\{0, \lambda(\epsilon)^{2}\right\}, n=1, \ldots, N$. Then

$$
\begin{aligned}
& \frac{(d-1)}{d} \int d \mathbf{k} g\left(\omega(\mathbf{k})^{2}\right)\left|F_{\epsilon}(\mathbf{k})\right|^{2} \\
& =\frac{g(0)}{\epsilon}-c_{\epsilon}^{2} g\left(\lambda(\epsilon)^{2}\right)+\sum_{n=1}^{N} \operatorname{Res}\left(\frac{g(z)}{z D(z)}, a_{n}\right),
\end{aligned}
$$

where $\operatorname{Res}\left(g(z) / z D(z), a_{n}\right)$ is the residue of $g(z) / z D(z)$ at $z=a_{n}$.

Proof: Similar to the proof of Lemma 3.4 in Ref. 16: one first notes that the lhs of (2.46) is written, via (2.44), as

$$
\frac{1}{2 \pi i} \int_{\omega_{0}^{2}}^{\infty} \frac{g(x)}{x}\left\{\frac{1}{D_{+}(x)}-\frac{1}{D_{-}(x)}\right\} d x .
$$

Then, by a contour integration of $g(z) / z D(z)$, one evaluates this integral, where Lemmas 2.3 and 2.4 are used.

Remark: Let $I_{\epsilon}(g)$ be the integral given by the lhs of (2.46) and consider the case $\omega_{0}=0$. Then (2.46) implies that, e.g., for $g \equiv 1$,

$$
\lim _{\epsilon \rightarrow 0} I_{c}(1)=\infty \text {. }
$$

On the other hand, for all $\mathbf{k} \in \mathbb{R}^{d} \backslash\{0\}$, we have

$$
\lim _{\epsilon \rightarrow 0} F_{\epsilon}(\mathbf{k})=i e\left[\hat{\rho}(\mathbf{k}) / \omega(\mathbf{k})^{2} m_{-}\left(\omega(\mathbf{k})^{2} ; 0\right)\right] \equiv F_{0}(\mathbf{k}) .
$$

If $\omega^{-2} \hat{\rho} \in L^{2}\left(\mathbb{R}^{d}\right)$, then $\left|F_{0}\right|^{2}$ is integrable [use (2.39)]. Hence, in this case, (2.47) implies that

$$
\lim _{\epsilon \rightarrow 0} I_{\epsilon}(1) \neq \frac{(d-1)}{d} \int d \mathbf{k}\left|F_{0}(\mathbf{k})\right|^{2},
$$

i.e., the limit $\epsilon \rightarrow 0$ does not commute with the integral in $I_{\epsilon}(1)$. This suggests that in the integral of a quantity containing $\left|F_{\epsilon}\right|^{2}$, we have to be careful about the interchange between the limit $\epsilon \rightarrow 0$ and the integral. The reason for this subtlety of the integral $I_{\epsilon}(g)$ with $\omega_{0}=0$ becomes transparent if we write $I_{\epsilon}(g)$ as follows:

$$
I_{\epsilon}(g)=\frac{1}{\pi} \int_{0}^{\infty} \frac{g(x) v(x)}{(\epsilon-x w(x))^{2}+x^{2} v(x)^{2}} d x
$$

where

$$
\begin{aligned}
& v(x)=\gamma_{d} \pi K_{0}(x), \\
& w(x)=m_{0}-\gamma_{d} \pi I_{0}(x) .
\end{aligned}
$$

We see that the right-hand side (rhs) of (2.48) is not an integral to which one can immediately apply the dominated convergence theorem to interchange the limit $\epsilon \rightarrow 0$ and the integral $\int d x$. An asymptotic estimate in $\epsilon$ for the integral of this type will be discussed in Appendix A.

For $h>0$, we define

$$
\begin{aligned}
T_{\mu \nu}^{(h)}\left(\mathbf{k}, \mathbf{k}^{\prime}\right)= & \delta_{\mu \nu} \delta\left(\mathbf{k}-\mathbf{k}^{\prime}\right) \\
& -i e \frac{\omega(\mathbf{k})^{2} F_{\epsilon}(\mathbf{k}) \hat{\rho}\left(\mathbf{k}^{\prime}\right) d_{\mu \nu}\left(\mathbf{k}^{\prime}\right)}{\omega(\mathbf{k})^{2}-\omega\left(\mathbf{k}^{\prime}\right)^{2}-i h}
\end{aligned}
$$

and

$$
\left(T_{\mu \nu}^{(h)} f\right)(\mathbf{k})=\int T_{\mu \nu}^{(h)}\left(\mathbf{k}, \mathbf{k}^{\prime}\right) f\left(\mathbf{k}^{\prime}\right) d \mathbf{k}^{\prime}
$$

In the case where $\omega_{0}>0$ and $\epsilon<\epsilon_{0}$, we also introduce the functions

$$
\phi_{\mu v}(\mathbf{k})=\frac{i e c_{\epsilon} \lambda(\epsilon)^{2} d_{\mu v}(\mathbf{k}) \hat{\rho}(\mathbf{k})}{\omega(\mathbf{k})^{2}-\lambda(\epsilon)^{2}}, \quad \mu, v=1, \ldots, d .
$$

We shall denote by $(\cdot, \cdot)$ the inner product of $L^{2}\left(\mathbb{R}^{d}\right)$.

Lemma 2.7: The operator $T_{\mu \nu}^{(h)}$ is a bounded linear operator on $L^{2}\left(\mathbb{R}^{d}\right)$ and the strong limit

$$
s-\lim _{h \rightarrow+0} T_{\mu v}^{(h)} \equiv T_{\mu v}
$$

exists. Further the following identities hold:

(i)

$$
T_{\alpha \beta}^{*} d_{\beta \mu} T_{\mu \nu}+\left(\phi_{\nu \beta}, \cdot\right) \phi_{\beta \alpha}=d_{\alpha \nu} I, \quad \alpha, v=1, \ldots, d .
$$

(ii)

$$
\begin{array}{r}
e_{\alpha}^{(r)} T_{\alpha \beta} d_{\beta \mu} T_{\mu \nu}^{*} e_{\nu}^{(s)}+\epsilon\left(F_{\varepsilon} e_{\alpha}^{(s)}, \cdot\right) F_{\epsilon} e_{\alpha}^{(r)}=\delta_{r s} I, \\
r, s=1, \ldots, d-1 .
\end{array}
$$

(iii)

$$
T_{\alpha \beta}^{*} d_{\beta \mu} F_{\epsilon}=c_{\epsilon} \phi_{\alpha \mu}, \quad \alpha, \mu=1, \ldots, d .
$$

(iv)

$$
e_{\mu}^{(r)} T_{\mu v} \phi_{v a}=\epsilon c_{\epsilon} F_{\epsilon} e_{\alpha}^{(r)}, \quad r=1, \ldots, d-1, \quad \alpha=1, \ldots, d .
$$

(v) In the case where $\omega_{0}>0$ and $\epsilon<\epsilon_{0}$, we have

$$
\left(\phi_{\mu \nu}, \phi_{\mu \alpha}\right)=\delta_{v \alpha}\left(1-\epsilon c_{\epsilon}^{2}\right), \quad \alpha, v=1, \ldots, d,
$$

and 
$e\left(\hat{\rho}, \phi_{\mu \nu}\right)=i c_{\epsilon}\left(\epsilon-m_{0} \lambda(\epsilon)^{2}\right) \delta_{\mu \nu}, \quad \mu, v=1, \ldots, d$. (vi)

$$
\left[\omega^{2}, T_{\mu \nu}\right]=-i e\left(\hat{\rho} d_{\mu v}, \cdot\right) \omega^{2} F_{\epsilon}, \quad \mu, v=1, \ldots, d .
$$

(vii)

$$
e T_{\mu \nu} \hat{\rho}=i \delta_{\mu \nu}\left(\epsilon-m_{0} \omega^{2}\right) F_{\epsilon}, \quad \mu, v=1, \ldots, d .
$$

Proof: Similar to the proof of Lemma 3.6 in Ref. 7.

The smeared time-zero fields are defined by

$$
\begin{aligned}
A_{\mu}(f) & \equiv \int d \mathbf{x} A_{\mu}(\mathrm{x}) f(\mathrm{x}) \\
& \equiv \frac{1}{\sqrt{2}}\left\{a_{r}\left(\omega^{-1 / 2} e_{\mu}^{(r) \hat{f}}\right)^{*}+a_{r}\left(\omega^{-1 / 2} e_{\mu}^{(r)} \hat{J f}\right)\right\},
\end{aligned}
$$

for $f$ satisfying $\omega^{-1 / 2} \hat{f} \in L^{2}\left(\mathbb{R}^{d}\right)$ and

$$
\begin{aligned}
\pi_{\mu}(f) & \equiv \int d \mathbf{x} \pi_{r}(\mathbf{x}) f(\mathbf{x}) \\
& =\frac{i}{\sqrt{2}}\left\{a_{r}\left(\omega^{1 / 2} e_{\mu}^{(r) \hat{f}}\right)^{*}-a_{r}\left(\omega^{1 / 2} e_{\mu}^{(r)} \hat{J f}\right)\right\},
\end{aligned}
$$

for $f$ satisfying $\omega^{1 / 2} \hat{f} \in L^{2}\left(\mathbb{R}^{d}\right)$, where $J$ denotes the complex conjugation operator:

$$
(J f)(\mathbf{k})=\overline{f(\mathbf{k})} \text {. }
$$

The Fourier transforms $\hat{A}_{\mu}$ and $\hat{\pi}_{\mu}$ of $A_{\mu}$ and $\pi_{\mu}$ are defined by

$$
\hat{A}_{\mu}(f)=A_{\mu}(\hat{f}), \quad \hat{\pi}_{\mu}(f)=\pi(\hat{f}),
$$

respectively.

We introduce the following operators:

$$
\begin{aligned}
b_{r}(f)= & \frac{1}{\sqrt{2}}\left\{\epsilon\left(f, \omega^{-1 / 2} F_{\epsilon} e_{\mu}^{(r)}\right) q_{\mu}+i\left(f, \omega^{1 / 2} F_{\epsilon} e_{\mu}^{(r)}\right) p_{\mu}\right. \\
& +\hat{A}_{\mu}\left(J\left(T_{\mu \nu}^{*} e_{\nu}^{(r)} \omega^{1 / 2} f\right)\right) \\
& \left.+i \hat{\pi}_{\mu}\left(J\left(T_{\mu \nu}^{*} e_{\nu}^{(r)} \omega^{-1 / 2} f\right)\right)\right\} \\
& r=1, \ldots, d-1, f \in L^{2}\left(\mathbb{R}^{d}\right) .
\end{aligned}
$$

In the case where $\omega_{0}>0$ and $\epsilon<\epsilon_{0}$, we also define

$$
\begin{aligned}
B_{\mu}= & \frac{\epsilon c_{\epsilon}}{\sqrt{2 \lambda(\epsilon)}} q_{\mu}+i c_{\epsilon} \sqrt{\frac{\lambda(\epsilon)}{2}} p_{\mu}+\sqrt{\frac{\lambda(\epsilon)}{2}} \hat{A}_{\nu}\left(\phi_{\nu \mu}\right) \\
& +\frac{i}{\sqrt{2 \lambda(\epsilon)}} \hat{\pi}_{\nu}\left(\phi_{\nu \mu}\right), \quad \mu=1, \ldots, d .
\end{aligned}
$$

The domain of $b_{r}(f)^{\#}$ and of $B_{\mu}^{\#}$ include $\mathscr{F}_{0}$, where $A^{\#}$ denotes either $A$ or $A^{*}$. Further, $b_{r}(f)^{\#}$ and $B_{\mu}^{\#}$ leave $\mathscr{F}_{0}$ invariant satisfying the commutation relations

$$
\begin{array}{r}
{\left[b_{r}(f), b_{s}(g)^{*}\right]=\delta_{r s}(f, g),\left[b_{r}(f), b_{s}(g)\right]=0,} \\
f, g \in L^{2}\left(\mathbb{R}^{d}\right), \quad r, s=1, \ldots, d-1, \\
{\left[B_{\mu}, B_{v}^{*}\right]=\delta_{\mu \nu}, \quad\left[B_{\mu}, B_{v}\right]=0, \quad \mu, v=1, \ldots, d,}
\end{array}
$$

on $\mathscr{F}_{0}$. We also have

$$
\begin{aligned}
& {\left[H, b_{r}(f)\right]=-b_{r}(\omega f), \quad \omega^{-1 / 2} f, \omega f \in L^{2}\left(\mathbb{R}^{d}\right),} \\
& {\left[H, B_{\mu}\right]=-\lambda(\epsilon) B_{\mu}, \quad \mu=1, \ldots, d,}
\end{aligned}
$$$$
\text { on } \mathscr{F}_{0} \cap D\left(H_{0}^{2}\right) \text {. }
$$

The main result in this subsection is the following.

Theorem 2.8: Suppose that in the case $\omega_{0}>0, \epsilon \neq \epsilon_{0}$. Let $f \in \mathscr{S}\left(\mathbb{R}^{d}\right)$ (the Schwartz space of rapidly decreasing $C^{\infty}$ functions on $\left.\mathbb{R}^{d}\right)$. Then, for all $t \in \mathbb{R}$ and $\mu=1, \ldots, d$,

$$
\begin{aligned}
& A_{\mu}(t, f)=(1 / \sqrt{2})\left\{b_{r}\left(\omega^{-1 / 2} e^{i t \omega} e_{\nu}^{(r)} T_{\mu v} \hat{f}\right)^{*}\right. \\
& \left.+b_{r}\left(\omega^{-1 / 2} e^{i t \omega} e_{v}^{(r)} T_{\mu v} \hat{f}\right)\right\} \\
& -[1 / \sqrt{2 \lambda(\epsilon)}]\left\{\left(\hat{J f}, \phi_{\mu \nu}\right) B_{\nu} e^{-i \lambda(\epsilon) t}\right. \\
& \left.+\left(\phi_{\mu \nu}, \hat{f}\right) B_{\nu}^{*} e^{i \lambda(\epsilon) t}\right\} \text {, } \\
& \pi_{\mu}(t, f)=(i / \sqrt{2})\left\{b_{r}\left(\omega^{1 / 2} e^{i t \omega} e_{v}^{(r)} T_{\mu v} \hat{f}\right)^{*}\right. \\
& \left.-b_{r}\left(\omega^{1 / 2} e^{i t \omega} e_{v}^{(r)} T_{\mu \nu} \hat{J f}\right)\right\} \\
& +i \sqrt{\lambda(\epsilon) / 2}\left\{\left(\hat{J f}, \phi_{\mu \nu}\right) B_{\nu} e^{-i \lambda(\epsilon) t}\right. \\
& \left.-\left(\phi_{\mu v}, \hat{f}\right) B_{v}^{*} e^{i \lambda(\epsilon) t}\right\} \text {, } \\
& q_{\mu}(t)=(1 / \sqrt{2})\left\{b_{r}\left(\omega^{1 / 2} e^{i t \omega} e_{\mu}^{(r)} F\right)^{*}+b_{r}\left(\omega^{1 / 2} e^{i t \omega} e_{\mu}^{(r)} F\right)\right\} \\
& +c_{\epsilon} \sqrt{\lambda(\epsilon) / 2}\left\{B_{\mu}^{*} e^{i \lambda(\epsilon) t}+B_{\mu} e^{-i \lambda(\epsilon) t}\right\} \text {, }
\end{aligned}
$$

$p_{\mu}(t)$

$$
\begin{aligned}
= & (i \epsilon / \sqrt{2})\left\{b_{r}\left(\omega^{-1 / 2} e^{i t \omega} e_{\mu}^{(r)} F\right)^{*}-b_{r}\left(\omega^{-1 / 2} e^{i t \omega} e_{\mu}^{(r)} F\right)\right\} \\
& +\left[i \epsilon c_{\epsilon} / \sqrt{2 \lambda(\epsilon)}\right]\left\{B_{\mu}^{*} e^{i \lambda(\epsilon) t}-B_{\mu} e^{-i \lambda(\epsilon) t}\right\},
\end{aligned}
$$

on $\mathscr{F}_{0}$.

The proof of this theorem is similar to that of Theorem 3.1 in Ref. 7 and hence is omitted.

Using Theorem 2.8, we can express $a_{r}(f)^{\#}$ in terms of $b_{r}(f)^{\#}$ and $B_{\mu}^{\#}$ :

$$
\begin{aligned}
a_{r}(f)^{*}= & b_{s}\left(T_{+}^{(s, r)} f\right)^{*}+b_{s}\left(J\left(T_{-}^{(s, r)} \tilde{f}\right)\right) \\
& -\left(\phi_{+, \mu}^{(r)}, f\right) B_{\mu}^{*}-\left(\phi_{-, \mu}^{(r)}, \tilde{f}\right) B_{\mu},
\end{aligned}
$$

where

$$
\tilde{f}(\mathbf{k})=f(-\mathbf{k})
$$

and

$T_{ \pm}^{(r, s)}=\frac{1}{2}\left\{\omega^{-1 / 2} e_{\nu}^{(r)} T_{\nu \mu} e_{\mu}^{(s)} \omega^{1 / 2} \pm \omega^{1 / 2} e_{v}^{(r)} T_{\nu \mu} e_{\mu}^{(s)} \omega^{-1 / 2}\right\}$

$\phi_{ \pm, \mu}^{(r)}=\frac{1}{2}\left\{\lambda(\epsilon)^{1 / 2} \omega^{-1 / 2} \pm \lambda(\epsilon)^{-1 / 2} \omega^{1 / 2}\right\} \phi_{\mu \nu} e_{\nu}^{(r)}$.

By direct computations, one can easily prove the following fact.

Lemma 2.9: For each $r, s=1, \ldots, d-1, T_{-}^{(r, s)}$ is a Hilbert-Schmidt operator on $L^{2}\left(\mathbb{R}^{d}\right)$ with the integral kernel $T_{-}^{(r, s)}\left(\mathbf{k}, \mathbf{k}^{\prime}\right)$ given by

$$
\begin{aligned}
T_{-}^{(r, s)}\left(\mathbf{k}, \mathbf{k}^{\prime}\right)= & e_{\mu}^{(r)}(\mathbf{k}) e_{\mu}^{(s)}\left(\mathbf{k}^{\prime}\right) \\
& \times \frac{i e \omega(\mathbf{k})^{2} F_{\epsilon}(\mathbf{k}) \hat{\rho}\left(\mathbf{k}^{\prime}\right)}{2 \sqrt{\omega(\mathbf{k}) \omega\left(\mathbf{k}^{\prime}\right)\left(\omega(\mathbf{k})+\omega\left(\mathbf{k}^{\prime}\right)\right)}} .
\end{aligned}
$$

\section{Spectra of the Hamiltonian} of $H$.

We next state results concerning the spectral properties

Lemma 2.10: (i) Let either $\omega_{0}=0$ or $\omega_{0}>0, \epsilon>\epsilon_{0}$. Then there exists a unique (up to constant multiples) vector $\Phi_{0}$ in $\mathscr{F}$ with $\left\|\Phi_{o}\right\|=1$ such that for all $r=1, \ldots, d-1$, and $f \in L^{2}\left(\mathbb{R}^{d}\right)$, 


$$
b_{r}(f) \Phi_{0}=0 .
$$

Further $\Phi_{0}$ is in the domain of every polynomial of $b_{r}(f)^{\# \text {, }}$ $r=1, \ldots, d-1, f \in L^{2}\left(\mathbb{R}^{d}\right)$.

(ii) Let $\omega_{0}>0$ and $\epsilon<\epsilon_{0}$. Then, there exists a unique (up to constant multiples) vector $\Psi_{0}$ in $\mathscr{F}$ with $\left\|\Psi_{0}\right\|=1$ such that for all $r=1, \ldots, d-1, \mu=1, \ldots, d$, and $f \in L^{2}\left(\mathbb{R}^{d}\right)$,

$$
b_{r}(f) \Psi_{0}=0, \quad B_{\mu} \Psi_{0}=0 .
$$

Further $\Psi_{0}$ is in the domain of every polynomial of $b_{r}(f)^{\# \text {, }}$ $r=1, \ldots, d-1, f \in L^{2}\left(\mathbb{R}^{d}\right)$ and $B=1, \mu=1, \ldots, d$.

Proof: Similar to the proof of Lemma 5.6 in Ref. 7 and of Theorem 4.3 in Ref. 16.

Let

$A_{\mu}=\left(\frac{m_{0} \epsilon}{4}\right)^{1 / 4} q_{\mu}+i\left(4 m_{0} \epsilon\right)^{-1 / 4} p_{\mu}, \quad \mu=1, \ldots, d$.

Then we have

$$
\left[A_{\mu}, A_{v}^{*}\right]=\delta_{\mu v}, \quad \mu, v=1, \ldots, d,
$$

on $C_{0}^{\infty}\left(\mathbb{R}^{d}\right)$. In terms of $A_{\mu}^{\#}$, the harmonic oscillator Hamiltonian

$$
h_{\epsilon}=\mathbf{p}^{2} / 2 m_{0}+(\epsilon / 2) \mathbf{q}^{2}
$$

can be written as

$$
h_{\mathrm{\epsilon}}=\sqrt{\frac{\epsilon}{m_{\mathrm{o}}}} A_{\mu}^{*} A_{\mu}+\frac{d}{2} \sqrt{\frac{\epsilon}{m_{\mathrm{o}}}} .
$$

Let

$$
\psi_{0}(\mathbf{q})=\left(\sqrt{m_{0} \epsilon} / \pi\right)^{d / 4} e^{-\sqrt{m_{0}} \boldsymbol{\epsilon}^{2} / 2},
$$

which is the normalized positive ground state function of $h_{\epsilon}$.

We denote by $\sigma(H)$ the spectrum of $H$ and set

$E_{0}=\inf \sigma(H) \geqslant 0$,

which is the ground state energy of $H$.

Theorem 2.11: (i) Let either $\omega_{0}=0$ or $\omega_{0}>0, \epsilon>\epsilon_{0}$. Then there exists a unitary transformation $U$ from $\mathscr{F}$ onto $\mathscr{F}_{\text {EM }}$ satisfying the following conditions:

(a) $U \Phi_{0}=\Omega_{0}$

(b) $U b_{r}(f) U^{-1}=a_{r}(f), r=1, \ldots, d-1, f \in L^{2}\left(\mathbb{R}^{d}\right)$,

(c) $U H U^{-1}=H_{F}+E_{0}$.

(ii) Let $\omega_{0}>0$ and $\epsilon<\epsilon_{0}$. Then there exists a unitary transformation $V$ from $\mathscr{F}$ onto itself satisfying the following conditions:

(a)

(b)

$$
\nu \Psi_{0}=\psi_{0} \otimes \Omega_{0}
$$

$$
\begin{aligned}
V b_{r}(f) V^{-1}= & a_{r}(f), \quad V B_{\mu} V^{-1}=A_{\mu}, \\
& r=1, \ldots, d-1, \mu=1, \ldots, d, f \in L^{2}\left(\mathbb{R}^{d}\right) ;
\end{aligned}
$$

(c)

$$
V H V^{-1}=\lambda(\epsilon) A_{\mu}^{*} A_{\mu}+H_{F}+E_{0} .
$$

Proof: Similar to the proof of Theorem 3.1 in Ref. 16.

Theorem 2.11 shows in particular that $\Phi_{0}$ (resp. $\Psi_{0}$ ) is the ground state of $H$ in the case where either $\omega_{0}=0$ or $\omega_{0}>0, \epsilon>\epsilon_{0}$ (resp. $\omega_{0}>0$ and $\epsilon<\epsilon_{0}$ ).

We denote by $\sigma_{p}(H)\left[\operatorname{resp} . \sigma_{\mathrm{ac}}(H), \sigma_{\mathrm{sc}}(H)\right]$ the point (resp. absolutely continuous, singular continuous) spectrum of $H$. The part (c) of Theorem 2.11 (i), (ii), and the well-known fact about the spectral properties of $H_{F}$ and the harmonic oscillator imply the following results about the spectral properties of $H$.

Theorem 2.12: (i) Let either $\omega_{0}=0$ or $\omega_{0}>0, \epsilon>\epsilon_{0}$. Then

$\sigma(H)=\left\{E_{0}\right\} \cup\left[E_{0}+\omega_{0}, \infty\right), \quad \sigma_{\mathrm{ac}}(H)=\left[E_{0}+\omega_{0}, \infty\right)$, $\sigma_{\mathrm{p}}(\mathrm{H})=\left\{\mathrm{E}_{0}\right\}, \quad \sigma_{\mathrm{sc}}(H)=\varnothing$,

where the multiplicity of the eigenvalue $E_{0}$ is 1 .

(ii) Let $\omega_{0}>0$ and $\epsilon<\epsilon_{0}$. Then

$$
\begin{aligned}
& \sigma(H)=\left\{E_{0}+n \lambda(\epsilon)\right\}_{n=0}^{\infty} \cup\left[E_{0}+\omega_{0}, \infty\right), \\
& \sigma_{\mathrm{ac}}(H)=\left[E_{0}+\omega_{0}, \infty\right), \\
& \sigma_{p}(H)=\left\{E_{0}+n \lambda(\epsilon)\right\}_{n=0}^{\infty}, \quad \sigma_{\mathrm{sc}}(H)=\varnothing,
\end{aligned}
$$

where the multiplicity of the eigenvalue $E_{0}$ is 1 .

Remarks: (i) In the case of Theorem 2.12 (ii), the eigenvalue $E_{0}+n \lambda(\epsilon)$ with $n \geqslant 1$ is degenerate with the multiplicity equal to the cardinal number of the set $\left\{\left(n_{1}, \ldots, n_{d}\right) \mid n_{1}+\cdots+n_{d}=n, n_{\mu} \geqslant 0, \mu=1, \ldots, d\right\}$. Further, by applying the Weierstrass preparation theorem, we can show that $\lambda(\epsilon)^{2}$ is analytic in $e^{2}$ in a neighborhood of the origin, having the Taylor expansion

$$
\begin{aligned}
\lambda(\epsilon)^{2}= & \frac{\epsilon}{m_{0}}-\frac{\epsilon(d-1)}{m_{0}^{2} d} \\
& \times\left(\int d \mathbf{k} \frac{\hat{\rho}(\mathbf{k})^{2}}{\omega(\mathbf{k})^{2}-\epsilon / m_{0}}\right) e^{2}+O\left(e^{4}\right),
\end{aligned}
$$

for $e^{2}$ sufficiently small.

(ii) Theorem 2.12 is interesting also from a point of view of perturbation theory of embedded eigenvalues. The unperturbed Hamiltonian $H_{0}$ given by (2.15) has infinitely many eigenvalues embedded in its continuous spectrum. Theorem 2.12(i) shows that in the case where $\omega_{0}=0$ or $\omega_{0}>0, \epsilon>\epsilon_{0}$, all the embedded eigenvalues of $H_{0}$, except for the least one, disappear under the perturbation $H-H_{0}$. This instability of embedded eigenvalues is related to the "resonance" of the harmonic oscillator atom modeled by $H$ (cf. Refs. 7 and 14 ). On the other hand, Theorem 2.12(ii) tells us that if $\omega_{0}>0$ and $\epsilon<\epsilon_{0}$, then the embedded eigenvalues of $H_{0}$ persist, changing their position in the continuous spectrum of $H$. Thus the stability of the embedded eigenvalues of $H_{0}$ depends on the strength of the parameters contained in $H$. This kind of phenomenon occurs also in other models (e.g., Ref. 15).

\section{The vacuum expectation values and the ground state energy}

In the last subsection we have seen that the ground state (vacuum) of $H$ exists. Hence we can construct the vacuum expectation values (VEVs) of the Heisenberg operators given by (2.16)-(2.19). It follows from Theorem 2.8, Lemma $2.10,(2.61)$ and (2.62) that all the $n$-point VEVs are determined by the two-point functions. We first consider the twopoint functions of the electron defined by 


$$
\begin{aligned}
& \left\langle q_{\mu}(t) q_{v}(s)\right\rangle_{M, \epsilon}=\left(\Omega, q_{\mu}(t) q_{v}(s) \Omega\right) \\
& \left\langle p_{\mu}(t) p_{\nu}(s)\right\rangle_{M, \epsilon}=\left(\Omega, p_{\mu}(t) p_{\mu}(s) \Omega\right), \quad t, s \in \mathbb{R},
\end{aligned}
$$

where $\Omega\left(=\Phi_{0}\right.$ or $\Psi_{0}$ ) denotes the ground state of $H$. By using (2.67), (2.68), and Lemma 2.10, we obtain the following formulas:

$$
\begin{aligned}
& \left\langle q_{\mu}(t) q_{\nu}(s)\right\rangle_{M, \epsilon} \\
& =\frac{\delta_{\mu v}}{2}\left\{\frac{(d-1)}{d} \int d \mathbf{k} \omega(\mathbf{k})\left|F_{\epsilon}(\mathbf{k})\right|^{2} e^{-i \omega(\mathbf{k})(t-s)}\right. \\
& \left.\quad+\lambda(\epsilon) c_{\epsilon}^{2} e^{-i \lambda(\epsilon)(t-s)}\right\} \\
& \left\langle p_{\mu}(t) p_{v}(s)\right\rangle_{M, \epsilon} \\
& =\frac{\delta_{\mu v}}{2}\left\{\frac{\epsilon^{2}(d-1)}{d} \int d \mathbf{k} \frac{\left|F_{\epsilon}(\mathbf{k})\right|^{2}}{\omega(\mathbf{k})} e^{-i \omega(\mathbf{k})(t-s)}\right. \\
& \left.\quad+\frac{\epsilon^{2} c_{\epsilon}^{2}}{\lambda(\epsilon)} e^{-i \lambda(\epsilon)(t-s)}\right\} .
\end{aligned}
$$

Using formula (2.44), we can rewrite (2.78) and (2.79) in simpler forms:

$$
\begin{aligned}
& \left\langle q_{\mu}(t) q_{\nu}(s)\right\rangle_{M, \epsilon} \\
& =\delta_{\mu \nu}\left\{\frac{1}{\pi} \int_{\omega_{0}}^{\infty} e^{-i(t-s) x} \operatorname{Im} D_{+}\left(x^{2}\right)^{-1} d x\right. \\
& \left.\quad+\frac{\lambda(\epsilon) c_{\epsilon}^{2}}{2} e^{-i \lambda(\epsilon)(t-s)}\right\}, \\
& \left\langle p_{\mu}(t) p_{\nu}(s)\right\rangle_{M, \epsilon} \\
& =\delta_{\mu \nu}\left\{\frac{\epsilon^{2}}{\pi} \int_{\omega_{0}}^{\infty} \frac{e^{-i(t-s) x}}{x^{2}} \operatorname{Im} D_{+}\left(x^{2}\right)^{-1} d x\right. \\
& \left.+\frac{\epsilon^{2} c_{\epsilon}^{2}}{2 \lambda(\epsilon)} e^{-i \lambda(\epsilon)(t-s)}\right\} .
\end{aligned}
$$

Hence, the VEVS of the potential and the kinetic energy of the electron are given by the following formulas:

$$
\begin{aligned}
\left\langle\frac{\epsilon}{2} \mathbf{q}(t)^{2}\right\rangle_{M, \epsilon}= & \frac{d}{\pi} \int_{\omega_{0}}^{\infty} \frac{\epsilon}{2} \operatorname{Im} D_{+}\left(x^{2}\right)^{-1} d x \\
& +\left[d \epsilon \lambda(\epsilon) c_{\epsilon}^{2}\right] / 4 \\
\left\langle\frac{\mathbf{p}(t)^{2}}{2 m_{0}}\right\rangle_{M, \epsilon}= & \frac{d \epsilon^{2}}{\pi} \int_{\omega_{0}}^{\infty} \frac{1}{2 m_{0} x^{2}} \operatorname{Im} D_{+}\left(x^{2}\right)^{-1} d x \\
& +\frac{d \epsilon^{2} c_{\epsilon}^{2}}{4 m_{0} \lambda(\epsilon)} .
\end{aligned}
$$

Let $\mathbf{v}(t)$ be the velocity of the electron:

$$
\mathbf{v}(t)=\frac{d \mathbf{q}(t)}{d t} .
$$

Then, in the same way as above, we have

$$
\begin{aligned}
\left\langle v_{\mu}(t) v_{\nu}(s)\right\rangle_{M, \epsilon} & \\
= & \delta_{\mu \nu}\left\{\frac{1}{\pi} \int_{\omega_{0}}^{\infty} x^{2} e^{-i(t-s) x} \operatorname{Im} D_{+}\left(x^{2}\right)^{-1} d x\right. \\
& \left.+\frac{\lambda(\epsilon)^{3} c_{\varepsilon}^{2}}{2} e^{-i \lambda(\epsilon)(t-s)}\right\} .
\end{aligned}
$$

In particular, we obtain

$$
\begin{aligned}
\left\langle\frac{m_{0}}{2} \mathrm{v}(t)^{2}\right\rangle_{M, \epsilon}= & \frac{d}{\pi} \int_{\omega_{0}}^{\infty} \frac{m_{0}}{2} x^{2} \operatorname{Im} D_{+}\left(x^{2}\right)^{-1} d x \\
& +\left[d m_{0} \lambda(\epsilon)^{3} c_{\epsilon}^{2}\right] / 4
\end{aligned}
$$

As is seen from the above formulas, in the case $\omega_{0}>0$ and $\epsilon<\epsilon_{0}$, the two-point functions $\left\langle q_{\mu}(t) q_{\mu}\right\rangle_{M, \epsilon}$, $\left\langle p_{\mu}(t) p_{\mu}\right\rangle_{M, \epsilon}$, and $\left\langle v_{\mu}(t) v_{\mu}\right\rangle_{M, \epsilon}$ do not decay as $t \rightarrow \pm \infty$ because of the oscillating part $\exp (-i \lambda(\epsilon) t)$. On the other hand, if $\omega_{0}=0$ or $\omega_{0}>0, \epsilon>\epsilon_{0}$, then we can show that under suitable conditions, they decay with a power-law, respectively (see Ref. 22).

Concerning the VEVs of the radiation field, we consider only the following one:

$$
\begin{array}{r}
\left\langle a_{r}(t, f)^{*} a_{s}(g)\right\rangle_{M, \epsilon} \equiv\left(\Omega, a_{r}(t, f)^{*} a_{s}(g) \Omega\right), \\
f, g \in L^{2}\left(\mathbb{R}^{d}\right), r, s=1, \ldots, d-1, t \in \mathbb{R},
\end{array}
$$

where

$$
a_{r}(t, f)=e^{i t H} a_{r}(f) e^{-i t H} .
$$

To represent $\left\langle a_{r}(t, f) * a_{s}(g)\right\rangle_{M, \varepsilon}$ explicitly, we introduce

$$
\begin{aligned}
f_{\epsilon}(t, x, y) \equiv & \frac{(d-1) e^{2}}{d} \int \frac{\omega(\mathbf{k})^{3}\left|F_{\epsilon}(\mathbf{k})\right|^{2} e^{-i t \omega(\mathbf{k})}}{(\omega(\mathbf{k})+x)(\omega(\mathbf{k})+y)} d \mathbf{k} \\
= & \frac{2 e^{2}}{\pi} \int_{\omega_{0}}^{\infty} \frac{w^{2} e^{-i t w}}{(w+x)(w+y)} \\
& \times \operatorname{Im} D_{+}\left(w^{2}\right)^{-1} d w, \quad x, y \geqslant 0
\end{aligned}
$$

and

$$
\psi_{ \pm}(\mathbf{k})=\theta\left(\omega_{0}\right) \theta\left(\epsilon_{0}-\epsilon\right) \frac{e c_{\epsilon} \lambda(\epsilon)^{3 / 2} \hat{\rho}(\mathbf{k})}{\lambda(\epsilon) \pm \omega(\mathbf{k})}
$$

where in the last equality in (2.89), we have used (2.44). Since $\left\langle a_{r}(t, f)^{*} a_{s}(g)\right\rangle_{M, \epsilon}$ defines a tempered distribution on $\mathbb{R}^{d} \times \mathbb{R}^{d}$, we may write symbolically

$$
\begin{aligned}
\left\langle a_{r}(t, f)^{*} a_{s}(g)\right\rangle_{M, \epsilon} & \\
= & \int d \mathbf{k}\left\langle a_{r}(t, \mathbf{k})^{*} a_{s}\left(\mathbf{k}^{\prime}\right)\right\rangle_{M, \epsilon} f(\mathbf{k}) \overline{g\left(\mathbf{k}^{\prime}\right)}, \\
& f, g \in \mathscr{S}\left(\mathbb{R}^{d}\right) .
\end{aligned}
$$

Lemma 2.13:

$$
\begin{aligned}
\left\langle a_{r}(t, \mathbf{k}) * a_{s}\left(\mathbf{k}^{\prime}\right)\right\rangle_{M, \epsilon} & \\
= & e_{\mu}^{(r)}(\mathbf{k}) e_{\mu}^{(s)}\left(\mathbf{k}^{\prime}\right) / 4 \sqrt{\omega(\mathbf{k}) \omega\left(\mathbf{k}^{\prime}\right)} \\
& \times\left\{\hat{\rho}(\mathbf{k}) \hat{\rho}\left(\mathbf{k}^{\prime}\right) f_{\epsilon}\left(t, \omega(\mathbf{k}), \omega\left(\mathbf{k}^{\prime}\right)\right)\right. \\
& \left.+\psi_{+}(\mathbf{k}) \psi_{+}\left(\mathbf{k}^{\prime}\right) e^{-i \lambda(\epsilon)}\right\} .
\end{aligned}
$$

Proof: We have from (2.69) and Lemma 2.10

$$
\begin{aligned}
\left\langle a_{r}(t, f)^{*} a_{s}(g)\right\rangle_{M, \epsilon}= & \left(\tilde{g}, W_{t}^{(s, r)} \tilde{f}\right) \\
& +\left(\tilde{g}, \phi_{-, \mu}^{(s)}\right)\left(\phi_{-, \mu}^{(r)}, \tilde{f}\right) e^{-i \lambda(\epsilon) t},
\end{aligned}
$$

where

$$
W_{r}^{(s, r)}=T_{-}^{(l, s)^{*}} e^{-i \omega t} T_{-}^{(l, r)} .
$$

Using (2.73), we can see that the operator $W_{t}^{(s, r)}$ is an integral operator (in fact, Hilbert-Schmidt) with the kernel 


$$
\begin{aligned}
W_{t}^{(s, r)}\left(\mathbf{k}, \mathbf{k}^{\prime}\right)= & \frac{e_{\mu}^{(s)}(\mathbf{k}) e_{\mu}^{(r)}\left(\mathbf{k}^{\prime}\right)}{4} \\
& \times\left\{\frac{\hat{\rho}(\mathbf{k}) \hat{\rho}\left(\mathbf{k}^{\prime}\right) f_{\epsilon}\left(t, \omega(\mathbf{k}), \omega\left(\mathbf{k}^{\prime}\right)\right)}{\sqrt{\omega(\mathbf{k}) \omega\left(\mathbf{k}^{\prime}\right)}}\right\} .
\end{aligned}
$$

Further, it follows from (2.72) that

$\phi_{-, \mu}^{(s)}\left(\mathbf{k}^{\prime}\right) \overline{\phi_{-, \mu}^{(r)}(\mathbf{k})}=\frac{1}{4} \frac{e_{\mu}^{(s)}\left(\mathbf{k}^{\prime}\right) e_{\mu}^{(r)}(\mathbf{k}) \psi_{+}\left(\mathbf{k}^{\prime}\right) \psi_{+}(\mathbf{k})}{\sqrt{\omega(\mathbf{k}) \omega\left(\mathbf{k}^{\prime}\right)}}$

Thus (2.92) follows.

Formula (2.92) gives an explicit representation for the VEV of the free Hamiltonian of the radiation field:

$$
\left\langle H_{F}\right\rangle_{M, \epsilon}=\frac{(d-1)}{4} \int d \mathbf{k}\left\{f_{\varepsilon}(\omega(\mathbf{k})) \hat{\rho}(\mathbf{k})^{2}+\psi_{+}(\mathbf{k})^{2}\right\},
$$

where

$$
f_{\epsilon}(x)=f_{\epsilon}(0, x, x) .
$$

We are now ready to obtain a formula for the ground state energy of $H$ :

Theorem 2.14:

$$
\begin{aligned}
E_{0}= & \frac{d}{\pi} \int_{\omega_{0}}^{\infty}\left(\frac{m_{0}}{2} x^{2}+\frac{\epsilon}{2}\right) \operatorname{Im} D_{+}\left(x^{2}\right)^{-1} d x \\
& +d / 4\left(m_{0} \lambda(\epsilon)^{2}+\epsilon\right) \lambda(\epsilon) c_{\epsilon}^{2} \\
& +\frac{(d-1)}{4} \int d \mathbf{k}\left\{f_{\epsilon}(\omega(\mathbf{k})) \hat{\rho}(\mathbf{k})^{2}+\psi_{+}(\mathbf{k})^{2}\right\} .
\end{aligned}
$$

Proof: We have

$E_{0}=(\Omega, H \Omega)$.

By (2.23), $H$ can be written as

$$
H=\left(m_{0} / 2\right) \mathbf{v}(0)^{2}+(\epsilon / 2) \mathbf{q}^{2}+H_{F} .
$$

Then, using (2.82), (2.86), and (2.93), we get (2.94).

\section{THE NO-BINDING LIMIT AND THE LONG-TIME ASYMPTOTICS IN THE GROUND STATE}

In this section we consider the no-binding limit $\epsilon \rightarrow 0$ of the model in terms of the VEVs and the long-time asymptotic behavior of the mean-square displacement of the "free" electron in the ground state.

\section{A. The no-binding limit}

We first note the following fact.

Lemma 3.1: (i) Let $M=0$. Suppose that

$$
\lim _{x \rightarrow 0} K_{0}(x) / x^{\gamma}>0
$$

with some constant $\gamma>0$. Then, for each $\mu=1, \ldots, d$, and $t, s \in \mathbf{R}$,

$\lim _{\epsilon \rightarrow 0}\left|\left\langle q_{\mu}(t) q_{\mu}(s)\right\rangle_{0, \varepsilon}\right|=\infty$.

(ii) Let $M>0$. Then, for each $\mu=1, \ldots, d, t, s \in \mathbb{R}$,

$\lim _{\epsilon \rightarrow 0}\left|\left\langle q_{\mu}(t) q_{\mu}(s)\right\rangle_{M, \epsilon}\right|=\infty$.

Proof: (i) We prove (3.2) only in the case $\tau=t-s>0$.
The case $t=s$ can be more easily treated. For each $\mu=1, \ldots, d$, we have from $(2.78)$

$$
\begin{aligned}
& \left|\left\langle q_{\mu}(t) q_{\mu}(s)\right\rangle_{0, \epsilon}\right| \\
& \quad \geqslant\left.\frac{(d-1)}{2 d}\left|\int d \mathbf{k} \omega(\mathbf{k})\right| F_{\epsilon}(\mathbf{k})\right|^{2} \cos \omega(\mathbf{k}) \tau \mid \\
& \quad \geqslant \frac{1}{2 \sqrt{2}} f_{\epsilon}\left(\frac{\pi}{4 \tau}\right)
\end{aligned}
$$

where

$$
f_{\epsilon}(a)=\frac{(d-1)}{d} \int_{\omega(\mathbf{k})<a} d \mathbf{k} \omega(\mathbf{k})\left|F_{\epsilon}(\mathbf{k})\right|^{2} .
$$

We can write

$$
f_{\epsilon}(a)=\frac{1}{\pi} \int_{0}^{a^{2}} d x \frac{\sqrt{x} v(x)}{(\epsilon-x w(x))^{2}+x^{2} v(x)^{2}},
$$

where $v$ and $w$ are defined by (2.49) and (2.50), respectively. Under condition (3.1), one can show [e.g., Ref. 23 (Sec. $5.15)]$ that

$$
w(x)-m=O\left(x^{\gamma}\right)(x \rightarrow 0),
$$

where $m$ is defined by (2.30). Hence, we can apply Proposition $\mathrm{A}$ in Appendix A to obtain

$$
\lim _{\epsilon \rightarrow 0} f_{\epsilon}(a)=\infty \text {. }
$$

Thus (3.2) follows.

(ii) Let

$$
R_{\epsilon}(t)=\int d \mathbf{k} \omega(\mathbf{k})\left|F_{\epsilon}(\mathbf{k})\right|^{2} e^{-i \omega(\mathbf{k}) t} .
$$

Then, by (2.78), we have for each $\mu=1, \ldots, d$,

$$
\begin{aligned}
& \left\langle q_{\mu}(t) q_{\mu}(s)\right\rangle_{M, \epsilon} \\
& \quad=\frac{1}{2}\left\{\frac{(d-1)}{d} R_{\epsilon}(t-s)+\lambda(\epsilon) c_{\epsilon}^{2} e^{-i \lambda(\epsilon)(t-s)}\right\} .
\end{aligned}
$$

By (2.39) and the asymptotic property of $D_{-}(x)$ as $x \rightarrow \omega_{0}^{2}$, we can show that

$$
|D(x-i 0)|^{2} \geqslant C\left(x^{2}+1\right), \quad x \geqslant \omega_{0}^{2},
$$

with a constant $C>0$ independent of $\epsilon>0$ sufficiently small. Hence, we have

$$
\omega(\mathbf{k})\left|F_{\epsilon}(\mathbf{k})\right|^{2} \leqslant e^{2} \omega(\mathbf{k})|\hat{\rho}(\mathbf{k})|^{2} / C\left(\omega(\mathbf{k})^{4}+1\right) .
$$

The rhs of this inequality is independent of $\epsilon$ sufficiently small and integrable with respect to $d \mathbf{k}$. Therefore, by the Lebesgue dominated convergence theorem, we obtain

$$
\lim _{\epsilon \rightarrow 0} R_{\epsilon}(t)=\int d \mathbf{k} \frac{e^{2}|\hat{\rho}(\mathbf{k})|^{2}}{\omega(\mathbf{k})^{3}\left|m_{-}\left(\omega(\mathbf{k})^{2} ; M\right)\right|^{2}} e^{-i \omega(t-s)} .
$$

We have from (2.31) and (2.45)

$$
\lambda(\epsilon) \sim \sqrt{\epsilon / m}, \quad c_{\epsilon}^{2} \sim 1 / \epsilon,
$$

as $\epsilon \rightarrow 0$, so that

$$
\lambda(\epsilon) c_{\epsilon}^{2} \rightarrow \infty
$$

as $\epsilon \rightarrow 0$. Applying this result and (3.6) to the rhs of (3.4), we obtain (3.3).

Lemma 3.1 shows that the no-binding limit does not 
exist for the VEVs of the electron position operator. This may be regarded as an indication of nonexistence of the ground state in the case $\epsilon=0$. We remark, however, that the two-point functions of the electron velocity have the nobinding limits (see subsection $C$ below).

We next consider the no-binding limit for the correlation functions of the position displacement operator of the electron

$$
\Delta \mathbf{q}(t)=\mathbf{q}(t)-\mathbf{q} .
$$

It follows from (2.67) that

$$
\begin{aligned}
\Delta \mathbf{q}_{\mu}(t)= & \sqrt{2} i\left\{b_{r}\left(\omega^{1 / 2} e_{\mu}^{(r)} F_{\epsilon} e^{i t \omega / 2} \sin t \omega / 2\right)^{*}\right. \\
& -b_{r}\left(\omega^{1 / 2} e_{\mu}^{(r)} F_{\epsilon} e^{i t \omega / 2} \sin t \omega / 2\right) \\
& +\sqrt{\lambda(\epsilon)} c_{\epsilon}\left(B_{\mu}^{*} e^{i t \lambda(\epsilon) / 2} \sin \lambda(\epsilon) t / 2\right. \\
& \left.\left.-B_{\mu} e^{-i t \lambda(\epsilon) / 2} \sin \lambda(\epsilon) t / 2\right)\right\} .
\end{aligned}
$$

Hence, the VEVs of $\Delta \mathbf{q}(t)$ can also be determined by the two-point functions

$$
\left\langle\Delta q_{\mu}(t) \Delta q_{v}(s)\right\rangle_{M, \epsilon}=\left(\Omega, \Delta q_{\mu}(t) \Delta q_{v}(s) \Omega\right),
$$

which are explicitly given by

$$
\begin{aligned}
\left\langle\Delta q_{\mu}(t) \Delta q_{v}(s)\right\rangle_{M, \epsilon} & \\
= & 2 \delta_{\mu \nu}\left\{\frac{(d-1)}{d} \int d \mathbf{k} \omega(\mathbf{k})\left|F_{\epsilon}(\mathbf{k})\right|^{2} e^{-i \omega(\mathbf{k})(t-s) / 2}\right. \\
& \times \sin \frac{\omega(\mathbf{k}) t}{2} \sin \frac{\omega(\mathbf{k}) s}{2} \\
& \left.+\lambda(\epsilon) c_{\epsilon}^{2} e^{-i \lambda(\epsilon)(t-s) / 2} \sin \frac{\lambda(\epsilon) t}{2} \sin \frac{\lambda(\epsilon) s}{2}\right\}
\end{aligned}
$$

Theorem 3.2: Let $M>0$. Then, for all $\mu$, $v=1, \ldots, d, t, s \in \mathbb{R}$,

$$
\lim _{\epsilon \rightarrow 0}\left\langle\Delta q_{\mu}(t) \Delta q_{v}(s)\right\rangle_{M, \epsilon} \equiv\left\langle\Delta q_{\mu}(t) \Delta q_{v}(s)\right\rangle_{M}
$$

exists and is given by

$$
\begin{aligned}
\left\langle\Delta q_{\mu}(t)\right. & \left.\Delta q_{\nu}(s)\right\rangle_{M} \\
= & 2 \delta_{\mu \nu} \frac{(d-1) e^{2}}{d} \int d \mathbf{k} \\
& \times \frac{\hat{\rho}(\mathbf{k})^{2}}{\omega(\mathbf{k})^{3}\left|m-\left(\omega(\mathbf{k})^{2} ; M\right)\right|^{2}} e^{-i \omega(\mathbf{k})(t-s)} \\
& \times \sin \frac{\omega(\mathbf{k}) t}{2} \sin \frac{\omega(\mathbf{k}) s}{2} .
\end{aligned}
$$

Proof: We can write

$$
\left\langle\Delta q_{\mu}(t) \Delta q_{v}(s)\right\rangle_{M, \epsilon}=2 \delta_{\mu v}\left\{\frac{(d-1)}{d} R_{\epsilon}(t, s)+r_{\epsilon}(t, s)\right\}
$$

with

$$
\begin{aligned}
R_{\epsilon}(t, s)= & \int d \mathbf{k} \omega(\mathbf{k})\left|F_{\epsilon}(\mathbf{k})\right|^{2} e^{-i \omega(\mathbf{k})(t-s)} \\
& \times \sin \frac{\omega(\mathbf{k}) t}{2} \sin \frac{\omega(\mathbf{k}) s}{2}
\end{aligned}
$$

and

$$
r_{\epsilon}(t, s)=\lambda(\epsilon) c_{\epsilon}^{2} e^{-i \lambda(\epsilon)(t-s) / 2} \sin \frac{\lambda(\epsilon) t}{2} \sin \frac{\lambda(\epsilon) s}{2} .
$$

Since $M>0$, we have

$$
\begin{aligned}
& \left|\omega(\mathbf{k}) F_{\epsilon}(\mathbf{k})^{2} e^{-i \omega(\mathbf{k})(t-s)} \sin \frac{\omega(\mathbf{k}) t}{2} \sin \frac{\omega(\mathbf{k}) s}{2}\right| \\
& \quad \leqslant C \omega(\mathbf{k}) \hat{\rho}(\mathbf{k})^{2},
\end{aligned}
$$

for all sufficiently small $\epsilon$ with a constant $C>0$ [cf. (3.5)]. The rhs of this inequality is integrable. Hence, by the Lebesgue dominated convergence theorem, we obtain

$$
\begin{aligned}
\lim _{\epsilon \rightarrow 0} R_{\epsilon}(t, s)= & e^{2} \int d \mathbf{k} \frac{\hat{\rho}(\mathbf{k})^{2}}{\omega(\mathbf{k})^{3} \mid m_{-}\left(\omega(\mathbf{k})^{2} ;\left.M\right|^{2}\right.} \\
& \times e^{-i \omega(\mathbf{k})(t-s)} \sin \frac{\omega(\mathbf{k}) t}{2} \sin \frac{\omega(\mathbf{k}) s}{2} .
\end{aligned}
$$

By (3.7) and the elementary fact that

$\sin x \sim x$

as $x \rightarrow 0$, we obtain

$$
\lim _{\epsilon \rightarrow 0} r_{\epsilon}(t, s)=0 .
$$

Thus (3.13) follows.

Remark: It is not so obvious whether or not formula (3.13) holds also for the case $M=0$. This problem is relatcd to the subtlety of the interchange between the limit $\epsilon \rightarrow 0$ and $\int d \mathbf{k}$ in integrals of quantities containing $\left|F_{\varepsilon}\right|^{2}$ (see Remark after Lemma 2.6). To avoid this difficulty, we shall define the two-point functions of $\Delta \mathrm{q}(t)$ for the case $M=0$ as the limit $M \rightarrow 0$ of those for the case $M>0$. See Theorem 3.4 below.

\section{B. The long-time behavior of the mean-square displacement}

Putting $t=s, \mu=v$, and taking the summation over $\mu$ in (3.13), we obtain an explicit formula for the mean-square displacement of the free electron in the ground state:

$$
\begin{aligned}
\left\langle\Delta \mathbf{q}(t)^{2}\right\rangle_{M}= & 2(d-1) e^{2} \int d \mathbf{k} \frac{\hat{\rho}(\mathbf{k})^{2}}{\omega(\mathbf{k})^{3}\left|m-\left(\omega(\mathbf{k})^{2} ; M\right)\right|^{2}} \\
& \times\left(\sin \frac{\omega(\mathbf{k}) t}{2}\right)^{2} .
\end{aligned}
$$

This formula immediately gives the following result.

Theorem 3.3: Let $M>0$. Then

$$
\sup _{t \in \mathbb{R}}\left\langle\Delta \mathbf{q}(t)^{2}\right\rangle_{M}<\infty \text {. }
$$

Proof: By (3.14) and (2.39), we have

$$
\begin{aligned}
\left\langle\Delta \mathbf{q}(t)^{2}\right\rangle_{M} & \leqslant 2(d-1) e^{2} \int d \mathbf{k} \frac{\hat{\rho}(\mathbf{k})^{2}}{\omega(\mathbf{k})^{3}\left|m_{-}\left(\omega(\mathbf{k})^{2} ; M\right)\right|^{2}} \\
& \leqslant \text { const } \int d \mathbf{k} \hat{\rho}(\mathbf{k})^{2}<\infty .
\end{aligned}
$$

Hence (3.15) follows.

Theorem 3.3 means that if the photons are massive, the electron does not diffuse in the ground state.

We next consider the case where the photons are massless, i.e., $M=0$. In this case, we want to define the meansquare displacement of the electron as $\lim _{M \rightarrow 0}\left\langle\Delta q(t)^{2}\right\rangle_{M}$. We set 


$$
m_{-}(x)=m_{-}(x ; 0)
$$

and

$$
E(\mathbf{k})=\omega_{1}(|\mathbf{k}|) \text {. }
$$

Theorem 3.4: For all $\mu, v=1, \ldots, d-1, t, s \in \mathbb{R}$, the limit

$$
\lim _{M \rightarrow 0}\left\langle\Delta q_{\mu}(t) \Delta q_{\nu}(s)\right\rangle_{M} \equiv\left\langle\Delta q_{\mu}(t) \Delta q_{\nu}(s)\right\rangle
$$

exists and is given by the rhs of (3.13) with $\omega(\mathbf{k})$ and $m_{-}\left(\omega(\mathbf{k})^{2} ; M\right)$ replaced by $E(\mathbf{k})$ and $m_{-}\left(E(\mathbf{k})^{2}\right)$, respectively. In particular, we have

$$
\begin{aligned}
\left\langle\Delta \mathbf{q}(t)^{2}\right\rangle= & 2(d-1) e^{2} \int d \mathbf{k} \frac{\hat{\rho}(\mathbf{k})^{2}}{E(\mathbf{k})^{3}\left|m_{-}\left(E(\mathbf{k})^{2}\right)\right|^{2}} \\
& \times\left(\sin \frac{E(\mathbf{k}) t}{2}\right)^{2} \cdot
\end{aligned}
$$

Proof: We have from the definition of $\omega(\mathbf{k})$ [see (2.4)] $\omega(\mathbf{k})>E(\mathbf{k})$

and

$$
\lim _{M \rightarrow 0} \omega(\mathbf{k})=E(\mathbf{k}) .
$$

By using (2.39) and the elementary inequality $|\sin x / x| \leqslant 1$, we have

$$
\frac{\hat{\rho}(\mathbf{k})^{2}}{\omega(\mathbf{k})^{3}\left|m_{-}\left(\omega(\mathbf{k})^{2} ; M\right)\right|^{2}}\left(\sin \frac{\omega(\mathbf{k}) t}{2}\right)^{2} \leqslant C \frac{\hat{\rho}(\mathbf{k})^{2}}{E(\mathbf{k})} t^{2},
$$

where $C>0$ is a constant independent of $M$ sufficiently small. The rhs of this inequality is integrable on $\mathbb{R}^{d}$. Further we can show that for all $x>0$,

$$
\lim _{M \rightarrow 0} m_{-}(x ; M)=m_{-}(x) .
$$

Thus we can apply the Lebesgue dominated convergence theorem to the integral on the rhs of (3.13) to obtain the desired result.

We note that formula (3.18) can be written as

$$
\left\langle\Delta \mathbf{q}(t)^{2}\right\rangle=\frac{4 d}{\pi} \int_{0}^{\infty} d x \frac{\sin ^{2} x t / 2}{x^{4}} \operatorname{Im} m_{+}\left(x^{2}\right)^{-1}
$$

[cf. (2.44)].

In the case $M=0$, the long-time asymptotics of $\left\langle\Delta \mathbf{q}(t)^{2}\right\rangle$ may depend on the infrared behavior of $\omega_{1}$ and of $\hat{\rho}$. We assume that

$$
\omega_{1}(k) \sim A_{0} k^{p}, \quad \omega_{1}^{\prime}(k) \sim p A_{0} k^{p-1}
$$

and

$$
\hat{\rho}(k) \sim \rho_{0} k^{r}
$$

as $k \rightarrow 0$ with some constants $A_{0}>0, \rho_{0}>0, p>0$, and $r \in \mathbb{R}$. Note that the condition $\omega^{-1} \hat{\rho} \in L^{2}\left(\mathbb{R}^{d}\right)$ implies that

$0<p<r+d / 2$.

Theorem 3.5: (i) If $0<p<(2 r+d) / 3$, then

$$
\sup _{\in \in \mathbf{R}}\left\langle\Delta \mathbf{q}(t)^{2}\right\rangle<\infty
$$

(ii) If $p=(2 r+d) / 3$, then

$\lim _{t \rightarrow \infty}\left\langle\Delta \mathbf{q}(t)^{2}\right\rangle=\infty$.
Further, for every $\epsilon \in(0,1)$, there exist positive constants $a$ and $b$ such that for all $t>0$,

$$
\left\langle\Delta \mathbf{q}(t)^{2}\right\rangle \leqslant a_{\epsilon} t^{\epsilon}+b_{\epsilon} .
$$

In particular, for all $\epsilon \in(0,1)$,

$$
\lim _{t \rightarrow \infty}\left\langle\Delta \mathbf{q}(t)^{2}\right\rangle / t^{\epsilon}=0 .
$$

(iii) If $(2 r+d) / 3<p<(2 r+d) / 2$, then

$$
\left\langle\Delta \mathbf{q}(t)^{2}\right\rangle \sim C(d, p) S(4-(2 r+d) / p) t^{(3 p-2 r-d) / p}
$$

as $t \rightarrow \infty$, where

$$
C(d, p)=2 d \gamma_{d} \rho_{0}^{2} / m^{2} p A_{0}^{(2 r+d) / p}
$$

and

$$
S(\alpha)=\int_{0}^{\infty} \frac{(\sin x / 2)^{2}}{x^{\alpha}}, \quad 1<\alpha<3 .
$$

Proof: We can write

$$
\left\langle\Delta \mathbf{q}(t)^{2}\right\rangle=2 d \gamma_{d} \int_{0}^{\infty} d x f(x) g(t x) d x,
$$

where

$$
f(x)=\frac{\eta(x)^{d-1} \eta^{\prime}(x) \hat{\rho}(\eta(x))^{2}}{x^{3}\left|m_{-}\left(x^{2}\right)\right|^{2}}
$$

and

$$
g(x)=(\sin x / 2)^{2} .
$$

We have

$$
f(x) \sim \frac{\rho_{0}^{2}}{m^{2} p A_{0}^{(2 r+d) / p}} \cdot \frac{1}{x^{(4 p-2 r-d) / p}}
$$

and

$$
g(x) \sim x^{2} / 4
$$

as $x \rightarrow 0$. Thus we can apply Proposition B in Appendix B to the integral (3.25) to obtain the desired results.

Let us apply Theorem 3.5 to the standard physical case: $d=3, p=1, r=0$. In this case, the assumption of part (ii) is satisfied. Hence, the electron diffuses very slowly.

Note that under the condition of Theorem 3.5(iii), the order of the divergence of $\left\langle\Delta \mathbf{q}(t)^{2}\right\rangle$ as $t \rightarrow \infty$ is less than 1 . Thus the transport of the electron in this case is not "regular" (cf. Ref. 4 for the terminology).

\section{The no-binding limit of the VEVs of the electron velocity}

In concluding this section, for a comparison, we consider the no-binding limit of the VEVs of the electron velocity. For $M>0$, we have from (2.85)

$$
\begin{aligned}
\left\langle v_{\mu}(t) v_{\nu}(s)\right\rangle_{M} \equiv & \lim _{c \rightarrow 0}\left\langle v_{\mu}(t) v_{\nu}(s)\right\rangle_{M, \epsilon} \\
= & \frac{\delta_{\mu \nu}(d-1) e^{2}}{2 d} \int d \mathbf{k} \\
& \times \frac{\hat{\rho}(\mathbf{k})^{2} e^{-i \omega(\mathbf{k})(t-s)}}{\omega(\mathbf{k})\left|m_{-}\left(\omega(\mathbf{k})^{2} ; M\right)\right|^{2}} .
\end{aligned}
$$

Hence, in contrast to the case of the VEVs of the electron position operator, the VEVs of the electron velocity have the no-binding limits. In the same way as in the proof of 
Theorem 3.4, we can show that for all $\mu, v=1, \ldots, d, t, s \in \mathbb{R}$, the massless limit

$$
\left\langle v_{\mu}(t) v_{v}(s)\right\rangle_{0} \equiv \lim _{M \rightarrow 0}\left\langle v_{\mu}(t) v_{\nu}(s)\right\rangle_{M}
$$

exists and is given by

$$
\begin{aligned}
\left\langle v_{\mu}(t) v_{v}(s)\right\rangle_{0}= & \frac{\delta_{\mu v}(d-1) e^{2}}{2 d} \int d \mathbf{k} \\
& \times \frac{\hat{\rho}(\mathbf{k})^{2} e^{-i E(\mathbf{k})(t-s)}}{E(\mathbf{k})\left|m_{-}\left(E(\mathbf{k})^{2}\right)\right|^{2}} .
\end{aligned}
$$

The Riemann-Lebesgue theorem applied to (3.26) and (3.28) implies that for all $M \geqslant 0$

$$
\lim _{|t| \rightarrow \infty}\left\langle v_{\mu}(t) v_{v}\right\rangle_{M}=0 .
$$

Remark: Formula (3.29) with $M=0$ can be elaborated: under (3.20) and (3.21) with some additional conditions, we have for each $\mu=1, \ldots, d$

$$
\left\langle v_{\mu}(t) v_{\mu}\right\rangle_{0} \sim \text { const } 1 / t^{(2 r+d-p) / p}
$$

as $t \rightarrow \infty$. This follows from a general theorem given in Ref. 22.

\section{FINITE TEMPERATURE CASES}

In this section we consider the case where the quantum system described by the model discussed in Sec. II is at a finite temperature. For this purpose we first construct an equilibrium (KMS) state of the model.

\section{A. A KMS state and correlation functions} 25).

We recall a definition of KMS states (e.g., Refs. 24 and

Definition 4. 1: Let $\mathscr{\Re}$ be a $C^{*}$ algebra and $\left\{\alpha_{t}\right\}_{t \in \mathbb{R}}$ be a one-parameter group of *automorphisms of $\mathfrak{A}$. A state $\langle\cdot\rangle$ of $\mathfrak{A}$ is said to be a KMS state at the inverse temperature $\beta>0$ with respect to $\left\{\alpha_{t}\right\}_{t \in \mathbb{R}}$ if there exists a norm-dense *subalgebra $\mathfrak{A}_{\alpha}$ in $\mathfrak{N}$ such that for all $A, B \in \mathfrak{I}_{\alpha}$, the mapping

$$
\mathbb{R} \ni t \rightarrow\left\langle A \alpha_{t}(B)\right\rangle
$$

can be extended to an analytic function in the strip $0<\operatorname{Im} t<\beta$ which is continuous on the boundary and satisfies

$$
\left.\left\langle A \alpha_{z}(B)\right\rangle\right|_{z=i \beta}=\langle B A\rangle .
$$

Let $\mathfrak{A}_{0}$ be the CCR *-algebra generated by the Weyl operators

$$
\begin{gathered}
W(\mathbf{f}, \mathbf{u}, \mathbf{v})=\exp i\left\{\frac{\mathbf{a}(\mathbf{f})^{*}+\mathbf{a}(\mathbf{f})}{\sqrt{2}}+\mathbf{u} \cdot \mathbf{q}+\mathbf{v} \cdot \mathbf{p}\right\}, \\
\mathbf{f}=\left(f_{1}, \ldots, f_{d-1}\right) \in W, \mathbf{u}, \mathbf{v} \in \mathbb{R}^{d},
\end{gathered}
$$

and $\mathfrak{A}$ be the $C^{*}$ algebra given by the completion of $\mathfrak{A}_{0}$ in the operator norm topology. For $t \in \mathbb{R}$, we define $\alpha_{t}: \mathfrak{U}_{0} \rightarrow \mathbb{B}(\mathscr{F})$ (the Banach algebra of all bounded linear operators on $\mathscr{F}$ ) by

$$
\alpha_{t}(W(\mathbf{f}, \mathbf{u}, \mathbf{v}))=e^{i t H} W(\mathbf{f}, \mathbf{u}, \mathbf{v}) e^{-i t H} .
$$

Lemma 4.2: The family $\left\{\alpha_{t}\right\}_{t \in \mathbb{R}}$ is a one-parameter group of *-automorphism of $\mathfrak{A}_{0}$.

Proof: The group property of $\alpha_{t}$ is obvious. The nontri- vial part to be proved is the surjectivity of $\alpha_{t}$. For each $r=1, \ldots, d-1$, we define $L_{r}: W \times \mathbb{R}^{d} \times \mathbb{R}^{d} \rightarrow L^{2}\left(\mathbb{R}^{d}\right)$ by

$$
\begin{aligned}
L_{r}(\mathrm{f}, \mathrm{u}, \mathrm{v})= & \sum_{s=1}^{d-1}\left(T_{+}^{(r, s)} f_{s}+J T_{-}^{(r, s)} \tilde{f}_{s}\right) \\
& +\left(\omega^{\mathrm{t} / 2} \mathrm{u} \cdot \mathrm{e}^{(r)}+i \epsilon \omega^{-1 / 2} \mathrm{v} \cdot \mathrm{e}^{(r)}\right) F_{\epsilon}
\end{aligned}
$$

and $M_{\mu}: W \times \mathbb{R}^{d} \times \mathbb{R}^{d} \rightarrow \mathbb{C}$ by

$$
\begin{aligned}
M_{\mu}(\mathrm{f}, \mathrm{u}, \mathrm{v})= & -\left(\phi_{+, \mu}^{(r)}, f_{r}\right)-\left(\tilde{f}_{r}, \phi_{-, \mu}^{(r)}\right) \\
& +\sqrt{\lambda(\epsilon)} c_{\epsilon} u_{\mu}+\left[i \epsilon c_{\epsilon} / \sqrt{\lambda(\epsilon)}\right] v_{\mu} .
\end{aligned}
$$

Then, by using $(2.65)-(2.68)$, we can write

$$
\begin{aligned}
W(\mathbf{f}, \mathrm{u}, \mathrm{v})= & \exp i(1 / \sqrt{2})\left\{b_{r}\left(L_{r}(\mathbf{f}, \mathbf{u}, \mathbf{v})\right)^{*}+b_{r}\left(L_{r}(\mathbf{f}, \mathrm{u}, \mathrm{v})\right)\right. \\
& \left.+M_{\mu}(\mathbf{f}, \mathbf{u}, \mathrm{v}) B_{\mu}^{*}+\overline{M_{\mu}(\mathrm{f}, \mathrm{u}, \mathrm{v})} B_{\mu}\right\} .
\end{aligned}
$$

Hence it follows from (2.63) and (2.64) that

$$
\begin{aligned}
& \alpha_{r}(W(\mathbf{f}, \mathbf{u}, \mathbf{v})) \\
& =\exp i(1 / \sqrt{2})\left\{b_{r}\left(e^{i t \omega} L_{r}(\mathbf{f}, \mathbf{u}, \mathbf{v})\right)^{*}+b_{r}\left(e^{i t \omega} L_{r}(\mathbf{f}, \mathbf{u}, \mathbf{v})\right)\right. \\
& \left.\quad+M_{\mu}(\mathbf{f}, \mathbf{u}, \mathbf{v}) B_{\mu}^{*} e^{i \lambda(\epsilon) t}+\overline{M_{\mu}(\mathbf{f}, \mathbf{u}, \mathbf{v})} B_{\mu} e^{-i t \lambda(\epsilon)}\right\}
\end{aligned}
$$

which, together with (2.59) and (2.60), implies that $\operatorname{Ran} \alpha_{t} \subset \mathfrak{U}_{0}$. This result combined with the group property of $\alpha_{t}$ gives the surjectivity of $\alpha_{t}$.

The above lemma implies that $\alpha_{t}$ extends uniquely to a one parameter group of *-automorphism of $\mathfrak{A}$. We denote the extension by the same symbol $\alpha_{i}$.

For each $\beta>0$ (the "inverse temperature"), we define a linear functional $\langle\cdot\rangle_{M, \epsilon}(\beta)$ on $\mathfrak{U}_{0}$ by

$$
\begin{aligned}
& \langle W(\mathbf{f}, \mathbf{u}, \mathbf{v})\rangle_{M, \epsilon}(\beta) \\
& =\exp -\frac{1}{4}\left\{\left(L_{r}(\mathbf{f}, \mathbf{u}, \mathbf{v}), \operatorname{coth}(\beta \omega / 2) L_{r}(\mathbf{f}, \mathbf{u}, \mathbf{v})\right)\right. \\
& \left.\quad+\operatorname{coth}[\beta \lambda(\epsilon) / 2]\left|M_{\mu}(\mathbf{f}, \mathbf{u}, \mathbf{v})\right|^{2}\right\},
\end{aligned}
$$

and by extending it to all elements in $\mathfrak{A}_{0}$ by linearity.

Theorem 4.3: The linear functional $\langle\cdot\rangle_{M, \epsilon}(\beta)$ is well defined and can be uniquely extended to a KMS state of $\mathcal{I}$ at the inverse temperature $\beta$ with respect to $\left\{\alpha_{t}\right\}_{t \in \mathbf{R}}$.

Proof: We prove Theorem 4.3 only in the case $M=0$. The other cases can be treated similarly. In the case $M=0$, the second term in $\{\cdot\}$ on the rhs of (4.7) does not appear. Let $\mathfrak{X}_{k^{*}}$ be the CCR $C^{*}$ algebra generated by the Weyl operators of the free quantized radiation field

$$
W_{F}(\mathbf{f})=\exp i(1 / \sqrt{2})\left\{\mathbf{a}(\mathbf{f})^{*}+\mathbf{a}(\mathbf{f})\right\}, \quad \mathbf{f} \in W,
$$

and $\left\{\alpha_{t}^{F}\right\}_{t \in \mathbf{R}}$ be the one-parameter group of $\mathfrak{A}_{F}$ defined by

$$
\alpha_{t}^{F}\left(W_{F}(\mathbf{f})\right)=e^{i t H_{F}} W_{F}(\mathbf{f}) e^{-i t H_{F}} .
$$

It is well known (e.g. Refs. 24 and 25 ) that the linear functional $\langle\cdot\rangle_{F}(\beta)$ on $\mathfrak{A}_{F}$ given by

$$
\left\langle W_{F}(\mathbf{f})\right\rangle_{F}(\beta) \equiv \exp \left\{-\frac{1}{4}\left(f_{r}, \operatorname{coth}(\beta \omega / 2) f_{r}\right)\right\}
$$

defines a KMS state of $\mathfrak{a}_{F}$ at the inverse temperature $\beta$ with respect to the one-parameter group $\left\{\alpha_{t}^{F}\right\}_{t \in \mathbb{R}}$. Using Theorem $2.11(\mathrm{i})$, we can easily see that

$$
U \mathfrak{W} U^{-1}=\mathfrak{\Re}_{F}, \quad U \alpha_{t}(A) U^{-1}=\alpha_{t}^{F}\left(U A U^{-1}\right)
$$

and

$$
\langle A\rangle_{M, \epsilon}(B)=\left\langle U A U^{-1}\right\rangle_{F} .
$$


These relations imply Theorem 4.3 .

The generating function for correlation functions of the harmonically bound electron is defined by

$Z_{\epsilon}^{(n)}\left(\mathbf{u}_{1}, \ldots, \mathbf{u}_{n} ; t_{1}, \ldots, t_{n} ; M, \beta\right)=\left\langle e^{i \mathbf{u}_{1} \cdot \mathbf{q}\left(t_{1}\right)} \cdots e^{i u_{n} \cdot \mathbf{q}\left(t_{n}\right)}\right\rangle_{M, \epsilon}(\beta)$.

By employing (2.67) and the Weyl relations, we can show that $Z_{\epsilon}^{(n)}$ has the following form:

$$
\begin{aligned}
Z_{\epsilon}^{(n)}\left(\mathbf{u}_{1}, \ldots, \mathbf{u}_{n} ; t_{1}, \ldots, t_{n} ; M, \beta\right) \\
=\exp \left\{-\frac{1}{2} \sum_{1<j<k<n} A_{\beta}\left(\mathbf{u}_{j}, \mathbf{u}_{k} ; t_{j}, t_{k}\right)\right. \\
\left.\quad-\frac{1}{4} \sum_{j=1}^{n} B_{\beta}\left(\mathrm{u}_{j}\right)\right\},
\end{aligned}
$$

where

$$
\begin{gathered}
A_{\beta}(\mathbf{u}, \mathbf{v} ; t, s)=\left(\omega^{1 / 2} F_{\epsilon} \mathbf{e}^{(r)} \cdot \mathbf{u}, S_{\beta}(t-s, \omega) \omega^{1 / 2} F_{\epsilon} \mathbf{e}^{(r)} \cdot \mathbf{v}\right) \\
+\lambda(\epsilon) c_{\epsilon}^{2} S_{\beta}(t-s, \lambda(\epsilon)) \mathbf{u} \cdot \mathbf{v}, \\
B_{\beta}(\mathbf{u})=\left(\omega^{1 / 2} F_{\epsilon} \mathbf{e}^{(r)} \cdot \mathbf{u}, S_{\beta}(0, \omega) \omega^{1 / 2} F_{\epsilon} \mathbf{e}^{(r)} \cdot \mathbf{u}\right) \\
+\lambda(\epsilon) c_{\epsilon}^{2} S_{\beta}(0, \lambda(\epsilon))|\mathbf{u}|^{2},
\end{gathered}
$$

with

$$
S_{\beta}(t, x)=\frac{e^{i t x}+e^{\beta x-i t x}}{e^{\beta x}-1}, \quad t \in \mathbb{R}, x \in \mathbb{R} \backslash\{0\} .
$$

Hence, $Z_{\epsilon}^{(n)}\left(\mathbf{u}_{1}, \ldots, \mathrm{u}_{n} ; t_{1}, \ldots . t_{n} ; M, \beta\right)$ is infinitely many times continuously differentible in $\left(\mathbf{u}_{1}, \ldots, \mathbf{u}_{n}\right)$. We define the $n$ point correlation functions of the harmonically bound electron by

$$
\begin{aligned}
& \left\langle q_{\mu_{1}}\left(t_{1}\right) \cdots q_{\mu_{n}}\left(t_{n}\right)\right\rangle_{M, \epsilon}(\beta) \\
& \left.\quad \equiv(-i)^{n} \frac{\partial^{n} \boldsymbol{Z}_{\epsilon}^{(n)}\left(\mathbf{u}_{1}, \ldots, \mathbf{u}_{n} ; t_{1}, \ldots, t_{n} ; M, \beta\right)}{\partial u_{1_{1}} \cdots \partial u_{n \mu_{n}}}\right|_{\mathbf{u}_{1}-\cdots-\mathbf{u}_{n}=0} .
\end{aligned}
$$

Then one can easily see that the $n$-point correlation functions are determined by the two-point correlation functions given by

$$
\begin{aligned}
& \left\langle q_{\mu}(t) q_{\nu}(s)\right\rangle_{M, \epsilon}(\beta) \\
& =\frac{1}{2}\left\{\left(\omega^{1 / 2} F_{\epsilon} e_{\mu}^{(r)}, S_{\beta}(t-s, \omega) \omega^{1 / 2} F_{\epsilon} \mathbf{e}_{\nu}^{(r)}\right)\right. \\
& \left.\quad+\delta_{\mu \nu} \lambda(\epsilon) c_{\epsilon}^{2} S_{\beta}(t-s, \lambda(\epsilon))\right\} .
\end{aligned}
$$

As in the case of VEVs, the two-point correlation function $\left\langle q_{\mu}(t) q_{\nu}(s)\right\rangle_{M, \epsilon}(\beta)$ can be rewritten as

$$
\begin{aligned}
\left\langle q_{\mu}(t) q_{\nu}(s)\right\rangle_{M, \epsilon}(\beta) & \\
= & \delta_{\mu \nu}\left\{\frac{1}{\pi} \int_{\omega_{0}}^{\infty} d x \frac{\cosh (\beta / 2-i(t-s)) x}{\sinh \beta x / 2}\right. \\
& \times \operatorname{Im} D_{+}\left(x^{2}\right)^{-1} \\
& \left.+\frac{\lambda(\epsilon) c_{\epsilon}^{2}}{2} \frac{\cosh (\beta / 2-i(t-s)) \lambda(\epsilon)}{\sinh \beta \lambda(\epsilon) / 2}\right\} .
\end{aligned}
$$

In particular, we have

$$
\begin{aligned}
\left\langle\mathbf{q}(t)^{2}\right\rangle_{M, \epsilon}(\beta)= & \frac{d}{\pi} \int_{\omega_{0}}^{\infty} d x \operatorname{coth} \frac{\beta x}{2} \operatorname{Im} D_{+}\left(x^{2}\right)^{-1} \\
& +\frac{d \lambda(\epsilon) c_{\epsilon}^{2}}{2} \operatorname{coth} \frac{\beta \lambda(\epsilon)}{2}
\end{aligned}
$$

Similarly we can define the correlation functions for the other Heisenberg operators and prove the following formulas:

$$
\begin{aligned}
\left\langle v_{\mu}(t) v_{\nu}(s)\right\rangle_{M, \epsilon}(\beta) & =\frac{\delta_{\mu v}}{2}\left\{\frac{(d-1)}{d} \int d \mathbf{k} \omega(\mathbf{k})^{3}\left|F_{\epsilon}(\mathbf{k})\right|^{2} S_{\beta}(t-s, \omega(\mathbf{k}))+\lambda(\epsilon)^{3} c_{\epsilon}^{2} S_{\beta}(t-s, \lambda(\epsilon))\right\} \\
& =\delta_{\mu v}\left\{\frac{1}{\pi} \int_{\omega_{0}}^{\infty} d x x^{2} \frac{\cosh (\beta / 2-i(t-s)) x}{\sinh \beta x / 2} \operatorname{Im} D_{+}\left(x^{2}\right)^{-1}+\frac{\lambda(\epsilon)^{3} c_{\epsilon}^{2}}{2} \frac{\cosh (\beta / 2-i(t-s)) \lambda(\epsilon)}{\sinh \beta \lambda(\epsilon) / 2}\right\} \\
\left\langle v(t)^{2}\right\rangle_{M, \epsilon}(\beta)=\frac{d}{\pi} \int_{\omega_{0}}^{\infty} d x x^{2} \operatorname{coth} \frac{\beta x}{2} \operatorname{Im} D_{+}\left(x^{2}\right)^{-1}+\frac{d \lambda(\epsilon)^{3} c_{\epsilon}^{2}}{2} \operatorname{coth} \frac{\beta \lambda(\epsilon)}{2} & \\
\left\langle p_{\mu}(t) p_{v}(s)\right\rangle_{M, \epsilon}(\beta) & =\frac{\delta_{\mu v}}{2}\left\{\frac{\epsilon^{2}(d-1) e^{2}}{d} \int d \mathbf{k} \frac{\hat{\rho}(\mathbf{k})^{2} e^{-i \omega(\mathbf{k})(t-s)}}{\left|D_{-}(\omega(\mathbf{k}))\right|^{2} \omega(\mathbf{k})} S_{\beta}(t-s, \omega(\mathbf{k}))+\frac{\epsilon^{2} c_{\epsilon}^{2}}{\lambda(\epsilon)} S_{\beta}(t-s, \lambda(\epsilon))\right\} \\
& =\delta_{\mu \nu}\left\{\frac{\epsilon^{2}}{\pi} \int_{\omega_{0}}^{\infty} d x \frac{\cosh (\beta / 2-i(t-s)) x}{x^{2} \sinh \beta x / 2} \operatorname{Im} D_{+}\left(x^{2}\right)^{-1}+\frac{\epsilon^{2} c_{\epsilon}^{2}}{2 \lambda(\epsilon)} \frac{\cosh (\beta / 2-i(t-s)) \lambda(\epsilon)}{\sinh \beta \lambda(\epsilon) / 2}\right\}
\end{aligned}
$$

$\left\langle\mathbf{p}(t)^{2}\right\rangle_{M, \epsilon}(\beta)=\frac{d \epsilon^{2}}{\pi} \int_{\omega_{0}}^{\infty} d x x^{-2} \operatorname{coth} \frac{\beta x}{2} \operatorname{Im} D_{+}\left(x^{2}\right)^{-1}+\frac{d \epsilon^{2} c_{\epsilon}^{2}}{2 \lambda(\epsilon)} \operatorname{coth} \frac{\beta \lambda(\epsilon)}{2}$

$\left\langle a_{r}(t, \mathbf{k})^{*} a_{s}\left(\mathbf{k}^{\prime}\right)\right\rangle_{M, \epsilon}(\beta)=e_{\mu}^{(r)}(\mathbf{k}) e_{\mu}^{(s)}\left(\mathbf{k}^{\prime}\right)\left\{\frac{e^{i \omega(\mathbf{k}) t}}{e^{\beta \omega(\mathbf{k})}-1} \delta\left(\mathbf{k}-\mathbf{k}^{\prime}\right)-\frac{i e}{2} \frac{1}{\sqrt{\omega(\mathbf{k}) \omega\left(\mathbf{k}^{\prime}\right)}\left(\omega\left(\mathbf{k}^{\prime}\right)-\omega(\mathbf{k})-i 0\right)}\right.$ 
where

$$
\begin{aligned}
& \left.\times\left[\frac{e^{i \omega\left(\mathbf{k}^{\prime}\right) t} \omega\left(\mathbf{k}^{\prime}\right)^{2} F_{\epsilon}\left(\mathbf{k}^{\prime}\right) \hat{\rho}(\mathbf{k})}{e^{\beta \omega\left(\mathbf{k}^{\prime}\right)}-1}+\frac{e^{i \omega(\mathbf{k}) t} \omega(\mathbf{k})^{2} F_{\epsilon}(\mathbf{k}) \hat{\rho}\left(\mathbf{k}^{\prime}\right)}{e^{\beta \omega(\hat{k})}-1}\right]\right\} \\
& +\frac{e_{\mu}^{(r)}(\mathbf{k}) e_{\mu}^{(s)}\left(\mathbf{k}^{\prime}\right)}{4 \sqrt{\omega(\mathbf{k}) \omega\left(\mathbf{k}^{\prime}\right)}}\left\{\left[g_{\epsilon, \beta}\left(t, \omega(\mathbf{k}), \omega\left(\mathbf{k}^{\prime}\right)\right)+f_{\epsilon, \beta}\left(t, \omega(\mathbf{k}), \omega\left(\mathbf{k}^{\prime}\right)\right)\right] \hat{\rho}(\mathbf{k}) \hat{\rho}\left(\mathbf{k}^{\prime}\right)\right. \\
& \left.+\psi_{+}(\mathbf{k}) \psi_{+}\left(\mathbf{k}^{\prime}\right) \frac{e^{-i t \lambda(\epsilon)} e^{\beta \lambda(\epsilon)}}{e^{\beta \lambda(\epsilon)}-1}+\psi_{-}(\mathbf{k}) \psi_{-}\left(\mathbf{k}^{\prime}\right) \frac{e^{i t \lambda(\epsilon)}}{e^{\beta \lambda(\epsilon)}-1}\right\}
\end{aligned}
$$

$$
\begin{aligned}
f_{\epsilon, \beta}(t, x, y) & \equiv \frac{(d-1)}{d} \int d \mathbf{k} \frac{e^{2} \omega(\mathbf{k})^{3} e^{(\beta-i t) \omega(\mathbf{k})}\left|F_{\epsilon}(\mathbf{k})\right|^{2}}{(\omega(\mathbf{k})+x)(\omega(\mathbf{k})+y)\left(e^{\beta \omega(\mathbf{k})}-1\right)} \\
& =\frac{2 e^{2}}{\pi} \int_{\omega_{0}}^{\infty} d w \frac{w^{2} e^{(\beta-i t) \omega}}{(w+x)(w+y)\left(e^{\beta \omega}-1\right)} \operatorname{Im} D_{+}\left(x^{2}\right)^{-1} \\
g_{\epsilon, \beta}(t, x, y) & \equiv \frac{(d-1)}{d} \int d \mathbf{k} \frac{e^{2} e^{i \omega(\mathbf{k})} \omega(\mathbf{k})^{3}\left|F_{\varepsilon}(\mathbf{k})\right|^{2}}{\omega(\mathbf{k})(\omega(\mathbf{k})-x+i 0)(\omega(\mathbf{k})-y-i 0)\left(e^{\beta(\omega)(\mathbf{k})}-1\right)} \\
& =\frac{2 e^{2}}{\pi} \int_{\omega_{0}}^{\infty} d w \frac{w^{2} e^{i \omega t}}{w^{2}(w-x+i 0)(w-y-i 0)\left(e^{\beta w}-1\right)} \operatorname{Im} D_{+}\left(x^{2}\right)^{-1}
\end{aligned}
$$

Remark: Formulas similar to (4.13) and (4.15) with $M=0$ have been given in Ref. 12 where a one-dimensional oscillator-dipole coupled to a three-dimensional radiation field is considered. The formulas obtained in this section include $d$-dimensional extensions of some formulas given in Ref. 12.

\section{B. The no-binding limit}

Proposition 4.4: Let $M>0$. Then, for all $\beta>0, \mu, v=1, \ldots, d, t, s \in \mathbb{R}$,

$$
\lim _{\epsilon \rightarrow 0} \epsilon\left\langle q_{\mu}(t) q_{\nu}(s)\right\rangle_{M, \epsilon}(\beta)=\delta_{\mu \nu} \beta-1 .
$$

Proof: Let

$$
\begin{aligned}
& L_{\epsilon}(t)=\frac{(d-1)}{d} \int d \mathbf{k} \omega(\mathbf{k})\left|F_{\epsilon}(\mathbf{k})\right|^{2} S_{\beta}(t, \omega(\mathbf{k})), \\
& l_{\epsilon}(t)=\lambda(\epsilon) c_{\epsilon}^{2} S_{\beta}(t, \lambda(\epsilon)),
\end{aligned}
$$

Then, by (4.11), we can write

$$
\left\langle q_{\mu}(t) q_{\nu}(s)\right\rangle_{M, \epsilon}(\beta)=\left(\delta_{\mu \nu} / 2\right)\left\{L_{\epsilon}(t-s)+l_{\epsilon}(t-s)\right\} .
$$

We have from (3.5)

$\omega(\mathbf{k})\left|F_{\epsilon}(\mathbf{k})\right|^{2} S_{\beta}(t, \omega(\mathbf{k})) \leqslant \frac{e^{2} \omega(\mathbf{k})|\hat{\rho}(\mathbf{k})|^{2}}{C\left(\omega(\mathbf{k})^{4}+1\right)}\left(1+\frac{2}{\beta \omega(\mathbf{k})}\right)$.

The rhs of this inequality is independent of $\epsilon$ sufficiently small and is integrable with respect to $d \mathbf{k}$. Therefore, by the Lebesgue dominated convergence theorem, we obtain

$$
\begin{aligned}
\lim _{\epsilon \rightarrow 0} L_{\epsilon}(t)= & \frac{e^{2}(d-1)}{d} \\
& \times \int d \mathbf{k} \frac{|\hat{\rho}(\mathbf{k})|^{2}}{\omega(\mathbf{k})^{3}\left|m_{-}\left(\omega(\mathbf{k})^{2} ; M\right)\right|^{2}} S_{\beta}(t, \omega(\mathbf{k})) .
\end{aligned}
$$

By (3.7) and

$$
S_{\beta}(t, x) \sim 2 / \beta x
$$

as $x \rightarrow 0$, we have

$$
l_{\epsilon}(t) \sim 2(\beta \epsilon)-1
$$

as $\epsilon \rightarrow 0$. Thus (4.22) follows.

Proposition 4.4 shows that the no-binding limit does not exist for the correlation functions of the harmonically bound electron. If we take a scaling for the position operator of the electron as

$$
\mathbf{q}(t, \epsilon) \equiv \sqrt{\epsilon \mathbf{q}}(t),
$$

then (4.22) implies that

$$
\lim _{\epsilon \rightarrow 0}\left\langle q_{\mu}(t, \epsilon) q_{v}(s, \epsilon)\right\rangle_{M, \epsilon}(\beta)=\delta_{\mu v} \beta^{-1}
$$

But this scaling limit is uninteresting, because it gives constant correlation functions.

We next consider the no-binding limit of the correlation functions of the displacement operator of the electron. We have from (4.11)

$$
\begin{aligned}
&\left\langle\Delta q_{\mu}(t) \Delta q_{\nu}(s)\right\rangle_{M, \epsilon}(\beta) \\
&= 2 \delta_{\mu \nu}\left\{\frac { ( d - 1 ) } { d } \left(\omega^{1 / 2} F_{\epsilon} \sin \frac{\omega t}{2}, S_{\beta}\left(\frac{t-s}{2}, \omega\right)\right.\right. \\
&\left.\times \omega^{1 / 2} F_{\epsilon} \sin \frac{\omega s}{2}\right)+\lambda(\epsilon) c_{\epsilon}^{2} S_{\beta}\left(\frac{t-s}{2}, \lambda(\epsilon)\right) \\
&\left.\times \sin \frac{\lambda(\epsilon) t}{2} \sin \frac{\lambda(\epsilon) s}{2}\right\} .
\end{aligned}
$$

Theorem 4.5: Let $M>0$. Then, for all $\beta>0, \mu, v=1, \ldots, d, t, s \in \mathbb{R}$, the limit

$$
\lim _{\epsilon \rightarrow 0}\left\langle\Delta q_{\mu}(t) \Delta q_{\nu}(s)\right\rangle_{M, \epsilon}(\beta) \equiv\left\langle\Delta q_{\mu}(t) \Delta q_{\nu}(s)\right\rangle_{M}(\beta)
$$

exists and is given by

$$
\begin{aligned}
& \left\langle\Delta q_{\mu}(t) \Delta q_{\nu}(u)\right\rangle_{M}(\beta) \\
& \quad=2 \delta_{\mu \nu}\left\{\frac{(d-1) e^{2}}{d} \int d \mathbf{k} \frac{\hat{\rho}(\mathbf{k})^{2}}{\omega(\mathbf{k})^{3}\left|m_{-}\left(\omega(\mathbf{k})^{2} ; M\right)\right|^{2}}\right.
\end{aligned}
$$




$$
\begin{aligned}
& \times S_{\beta}\left(\frac{t-s}{2}, \omega(\mathbf{k})\right) \sin \frac{\omega(\mathbf{k}) t}{2} \sin \frac{\omega(\mathbf{k}) s}{2} \\
& \left.+\frac{t s}{2 \beta m}\right\} .
\end{aligned}
$$

Proof: Similar to the proof of Theorem 3.4.

Remark: Note that in the case of finite temperatures, the contribution of the quasioscillator mode $B_{\mu}$ persists in the no-binding limit, producing the second term on the rhs of (4.25).

\section{The long-time asymptotics of the mean-square displacement}

By (4.25), the mean-square displacement of the electron at the finite temperature $\beta^{-1}$ is given by

$$
\begin{aligned}
\left\langle\Delta \mathbf{q}(t)^{2}\right\rangle_{M}(\beta) & \\
= & 2(d-1) e^{2} \int d \mathbf{k} \frac{\hat{\rho}(\mathbf{k})^{2}}{\omega(\mathbf{k})^{3}\left|m-\left(\omega(\mathbf{k})^{2} ; M\right)\right|^{2}} \\
& \times \operatorname{coth} \frac{\beta \omega(\mathbf{k})}{2}\left(\sin \frac{\omega(\mathbf{k}) t}{2}\right)^{2}+\frac{d}{\beta m} t^{2} .
\end{aligned}
$$

Theorem 4.6: Let $M>0$. Then

$$
\left\langle\Delta \mathbf{q}(t)^{2}\right\rangle_{M}(\beta) \sim(d / \beta m) t^{2}
$$

as $t \rightarrow \infty$.

Proof: The first term on the rhs of (4.26) is bounded uniformly in $t \in \mathbb{R}$ (cf. the proof of Theorem 3.3). Hence (4.27) follows.

Theorem 4.6 shows that in the case of finite temperatures, in contrast to the case of the zero temperature (Theorem 3.3), the electron diffuses with an infinite diffusion constant even in the case where the photons are massive. less.

We next consider the case where the photons are mass-

Theorem 4.7: For all $\beta>0, t \in \mathbb{R}$,

$$
\lim _{M \rightarrow 0}\left\langle\Delta \mathbf{q}(t)^{2}\right\rangle_{M}(\beta) \equiv\left\langle\Delta \mathbf{q}(t)^{2}\right\rangle(\beta)
$$

exists and is given by

$$
\left\langle\Delta \mathbf{q}(t)^{2}\right\rangle(\beta)=G_{\beta}(t)+(d / \beta m) t^{2},
$$

where

$$
\begin{aligned}
G_{\beta}(t)= & 2(d-1) e^{2} \int d \mathbf{k} \frac{\hat{\rho}(\mathbf{k})^{2}}{E(\mathbf{k})^{3}\left|m_{-}\left(E(\mathbf{k})^{2}\right)\right|^{2}} \\
& \times \operatorname{coth} \frac{\beta E(\mathbf{k})}{2}\left(\sin \frac{E(\mathbf{k}) t}{2}\right)^{2} .
\end{aligned}
$$

Proof: Similar to the proof of Theorem 3.4.

We note that $G_{\beta}$ can be written as

$$
G_{\beta}(t)=\left\langle\Delta \mathbf{q}(t)^{2}\right\rangle+H_{\beta}(t),
$$

where $\left\langle\Delta \mathbf{q}(t)^{2}\right\rangle$ is the mean-square displacement of the electron in the ground state [see (3.18)] and

$H_{\beta}(t)$

$$
\begin{aligned}
= & 4(d-1) e^{2} \int d \mathbf{k} \frac{|\hat{\rho}(\mathbf{k})|^{2}}{E(\mathbf{k})^{3}\left|m_{-}\left(E(\mathbf{k})^{2}\right)\right|^{2}\left(e^{\beta E(\mathbf{k})}-1\right)} \\
& \times\left(\sin \frac{t E(\mathbf{k})}{2}\right)^{2}
\end{aligned}
$$

Lemma 4.8: Assume (3.20) and (3.21). (i) If $0<p<(2 r+d) / 4$, then

$$
\sup _{t \in \mathbb{R}} H_{\beta}(t)<\infty \text {. }
$$

(ii) If $p=(2 r+d) / 4$, then

$\lim _{t \rightarrow \infty} H_{\beta}(t)=\infty$.

Further, for every $\epsilon \in(0,1)$, there exist positive constants $a$ and $b$ such that

$$
H_{\beta}(t) \leqslant a t^{\epsilon}+b .
$$

In particular, for every $\epsilon \in(0,1)$,

$$
\lim _{t \rightarrow \infty} H_{\beta}(t) / t^{\epsilon}=0 .
$$

(iii) If $(2 r+d) / 4<p<(2 r+d) / 2$, then

$$
H_{\beta}(t) \sim \frac{2 C(d, p)}{\beta} S\left(5-\frac{2 r+d}{p}\right) t^{(4 p-2 r-d) / p} .
$$

Proof: Similar to the proof of Theorem 3.5.

Combining Lemma 4.8 and Theorem 3.5, we obtain the following results about the asymptotic behavior of $\left\langle\Delta \mathbf{q}(t)^{2}\right\rangle(\beta)$ :

Theorem 4.9: Assume (3.20) and (3.21). (i) If $0<p<(2 r+d) / 4$, then

$$
\left\langle\Delta \mathbf{q}(t)^{2}\right\rangle(\beta) \sim(d / \beta m) t^{2}+O(1)
$$

as $t \rightarrow \infty$.

(ii) If $p=(2 r+d) / 4$, then for all $\epsilon \in(0,1)$

$$
\left\langle\Delta \mathbf{q}(t)^{2}\right\rangle(\beta) \sim(d / \beta m) t^{2}+o\left(t^{\epsilon}\right)
$$

as $t \rightarrow \infty$.

(iii) If $(2 r+d) / 4<p<(2 r+d) / 3$, then

$$
\begin{aligned}
\left\langle\Delta \mathbf{q}(t)^{2}\right\rangle(\beta) \sim & \frac{d}{\beta m} t^{2}+\frac{2 C(d, p) S(5-(2 r+d) / p)}{\beta} \\
& \times t^{(4 p-2 r-d) / p}+O(1)
\end{aligned}
$$

as $t \rightarrow \infty$.

(iv) If $p=(2 r+d) / 3$, then for all $\epsilon \in(0,1)$

$$
\begin{aligned}
\left\langle\Delta \mathbf{q}(t)^{2}\right\rangle(\beta) \sim & \frac{d}{\beta m} t^{2}+\frac{2 C(d, p) S(5-(2 r+d) / p)}{\beta} \\
& \times t^{(4 p-2 r-d) / p}+o\left(t^{\epsilon}\right)
\end{aligned}
$$

as $t \rightarrow \infty$.

(v) If $(2 r+d) / 3<p<(2 r+d) / 2$, then

$$
\begin{aligned}
\left\langle\Delta \mathbf{q}(t)^{2}\right\rangle(\beta) \sim & \frac{d}{\beta m} t^{2}+\frac{2 C(d, p) S(5-(2 r+d) / p)}{\beta} \\
& \times t^{(4 p-2 r-d) / p}+C(d, p) S\left(4-\frac{2 r+d}{p}\right) \\
& \times t^{(3 p-2 r-d) / p}+o\left(t^{(3 p-2 r-d) / p}\right)
\end{aligned}
$$

as $t \rightarrow \infty$.

Theorem 4.9 tells us that the electron diffuses with an infinite diffusion constant and the leading order $(=2)$ in the asymptotic expansion in $t$ of the mean-square displacement is independent of the space dimension $d$ and on the infrared behavior of $\omega_{1}$ and $\hat{\rho}$. The coefficient $d / \beta m$ of the leading term in each of the asymptotic expansions of $\left\langle\Delta \mathbf{q}(t)^{2}\right\rangle(\beta)$ has a physical meaning: We have from (4.18) 


$$
\left\langle\mathbf{p}(t)^{2}\right\rangle_{M}(\beta) \equiv \lim _{\epsilon \rightarrow 0}\left\langle\mathbf{p}(t)^{2}\right\rangle_{M, \epsilon}(\beta)=m d / \beta
$$

This formula carries over to the case $M=0$. Thus for all $M \geqslant 0$ and $\beta>0$ we have

$$
\left\langle\Delta \mathbf{q}(t)^{2}\right\rangle_{M}(\beta) \sim\left[\left\langle\mathbf{p}^{2}\right\rangle_{M}(\beta) / m^{2}\right] t^{2}
$$

as $t \rightarrow \infty$. This may be interpreted as showing that, in the asymptotic region $t \sim \infty$, the electron obeys the free motion in the sense of the mean value with the renormalized mass $m$. To elaborate this aspect, let us consider the no-binding limit of the velocity correlation functions. Let $M>0$ first. Then we have from $(4.14)$

$$
\begin{aligned}
\left\langle v_{\mu}(t) v_{\nu}\right\rangle_{M}(\beta) \equiv & \lim _{\epsilon \rightarrow 0}\left\langle v_{\mu}(t) v_{\nu}\right\rangle_{M, \epsilon}(\beta) \\
= & \frac{\delta_{\mu v}}{2}\left\{\frac{(d-1) e^{2}}{d} \int d \mathbf{k}\right. \\
& \left.\times \frac{\hat{\rho}(\mathbf{k})^{2} S_{\beta}(t, \omega(\mathbf{k}))}{\omega(\mathbf{k})\left|m_{-}\left(\omega(\mathbf{k})^{2} ; M\right)\right|^{2}}+\frac{2}{\beta m}\right\} .
\end{aligned}
$$

This formula carries over to the case $M=0$. By the Riemann-Lebesgue theorem, we have for each $\mu=1, \ldots, d, M \geqslant 0, \beta>0$,

$$
\lim _{|t| \rightarrow \infty}\left\langle v_{\mu}(t) v_{\mu}\right\rangle_{M}(\beta)=1 / \beta m .
$$

Thus, in the finite temperature cases, in contrast to the case of the zero temperature (the ground state), the velocity correlation does not vanish at $|t|=\infty$. Formula (4.35) also tells us that the mean value of the kinetic energy in the $\mu$ direction with the renormalized mass $m$ is given by

$$
\begin{aligned}
\left\langle\frac{m v_{\mu}(t)^{2}}{2}\right\rangle_{M}(\beta)= & \frac{1}{2 \beta}+\frac{m(d-1) e^{2}}{4 d} \\
& \times \int d \mathbf{k} \frac{\hat{\rho}(\mathbf{k})^{2} \operatorname{coth} \beta \omega(\mathbf{k}) / 2}{\omega(\mathbf{k})\left|m_{-}\left(\omega(\mathbf{k})^{2} ; M\right)\right|^{2}} .
\end{aligned}
$$

We note that the first term on the rhs of (4.37) is just equal to the mean value of the kinetic energy of one degree in an equilibrium ideal gas at the temperature $\beta^{-1}$. The second term on the rhs of (4.37) is regarded as the effect of the radiation field on the kinetic energy of the electron.

\section{ACKNOWLEDGMENT}

This work is supported by the Grant-In-Aid 02740094 for science research from the Ministry of Education, Japan.

\section{APPENDIX A: AN ESTIMATE FOR AN INTEGRAL}

Let $0<R<\infty$ be fixed and $\epsilon>0$. Let $v(x)$ be a continuous function on $[0, R]$ such that and

$$
v(x)>0, \text { for all } x \in(0, R]
$$

$$
\lim _{x \rightarrow 0} v(x) / x^{\alpha}>0
$$

exists with some $\alpha>0$. Further let $w(x)$ be a continuous function on $[0, R]$ such that

$$
|w(x)-1|=O\left(x^{\alpha}\right)(x \rightarrow 0) .
$$

Then, for each $\epsilon>0$ and $\lambda>-1$, we define the integral

$$
I(\epsilon)=\int_{0}^{R} \frac{x^{\lambda} v(x)}{(\epsilon-x w(x))^{2}+x^{2} v(x)^{2}} d x .
$$

The purpose of this appendix is to give an estimate in $\epsilon$ of $I(\epsilon)$

Proposition A: Let $v, w$ be as above. Then, for all sufficiently small $\epsilon>0$,

$$
C_{1} \epsilon^{\lambda-1} \leqslant I(\epsilon) \leqslant C_{2}\left(1+\epsilon^{\lambda-1-\alpha}\right)
$$

with constants $C_{j}>0, j=1,2$. In particular, if $-1<\lambda<1$, then

$$
\lim _{\epsilon \rightarrow 0} I(\epsilon)=\infty \text {. }
$$

Proof: Conditions (A1) and (A2) imply that

$$
c_{1} \leqslant v(x) / x^{\alpha} \leqslant c_{2}<\infty, \quad x \in(0, R]
$$

with positive constants $c_{1}$ and $c_{2}$. Hence, we have

$$
I(\epsilon) \geqslant c_{1} \int_{0}^{R} \frac{x^{\lambda+\alpha}}{(\epsilon-x w(x))^{2}+c_{2}^{2} x^{2(1+\alpha)}} d x .
$$

By the change of variable $x=\epsilon t$, we obtain

$$
I(\epsilon) \geqslant c_{1} \epsilon^{\lambda+\alpha-1} J(\epsilon),
$$

with

$$
J(\epsilon)=\int_{0}^{R / \epsilon} \frac{t^{\lambda+\alpha}}{(1-t w(\epsilon t))^{2}+c_{2}^{2} t^{2(1+\alpha)} \epsilon^{2 \alpha}} d t .
$$

Let $a>0$ be fixed and

$$
\epsilon<R /(1+a) \text {. }
$$

Then we have $[1,1+a] \subset[0, R / \epsilon]$. Hence

$$
\begin{aligned}
J(\epsilon) & >\int_{1}^{1+\alpha} \frac{t^{\lambda+\alpha}}{(1-t w(\epsilon t))^{2}+c_{2}^{2} t^{2(1+\alpha)} \epsilon^{2 \alpha}} d t \\
& \geqslant L(\epsilon)
\end{aligned}
$$

where

$$
L(\epsilon)=\int_{1}^{1+a} \frac{1}{(1-t w(\epsilon t))^{2}+c_{3} \epsilon^{2 a}} d t
$$

with $c_{3}=c_{2}^{2}(1+a)^{2(1+\alpha)}$. Further, by the change of variable $t=1+\epsilon^{\alpha} s$ and by using (A3), we see that

$$
L(\epsilon) \geqslant \epsilon^{-\alpha} N(\epsilon),
$$

where

$$
N(\epsilon)=\int_{0}^{a / \epsilon^{\alpha}} \frac{1}{\left(s+c_{4}\right)^{2}+c_{3}} d s
$$

with a positive constant $c_{4}$. Combining (A7)-(A9), we abtain

$$
I(\epsilon) \geqslant c_{1} \epsilon^{\lambda-1} N(\epsilon)
$$

Since

$$
0<\lim _{\epsilon \rightarrow 0} N(\epsilon)=\int_{0}^{\infty} \frac{1}{\left(s+c_{4}\right)^{2}+c_{3}} d s<+\infty,
$$

the first inequality in (A5) follows.

Let $c_{j}, j=1,2$, be as in (A6). Then we have, via the change of variable $x=\epsilon t$, 


$$
I(\epsilon)<c_{2} \epsilon^{\lambda-1+\alpha} Q(\epsilon),
$$

where

$$
Q(\epsilon)=\int_{0}^{R / \epsilon} \frac{t^{\lambda+\alpha}}{(1-t w(\epsilon t))^{2}+c_{1}^{2} \epsilon^{2 \alpha} t^{2+2 \alpha}} d t .
$$

Taking $\delta>0$ to be sufficiently small and dividing the integral interval into two parts $(0, \delta]$ and $(\delta, R / \epsilon]$, we can show that

$$
Q(\epsilon) \leqslant c_{3}+c_{4} \epsilon^{1-\alpha-\lambda}+c_{5} \epsilon^{-2 \alpha}
$$

with positive constants $c_{j}, j=3,4,5$. Thus the second inequality in (A5) follows.

Remark: Let $W_{\epsilon}(x)$ be the integrand of the integral (A4). Then we have for all $x \in(0, R]$

$$
\lim _{\epsilon \rightarrow 0} W_{\epsilon}(x) \equiv W_{0}(x)=\frac{v(x)}{x^{2-\lambda}\left(w(x)^{2}+v(x)^{2}\right)} .
$$

We see that if $1-\alpha<\lambda$, then $W_{0}$ is integrable on $(0, R]$ with respect to $d x$. Thus the above proposition shows that for $1-\alpha<\lambda<1$, the limit $\epsilon \rightarrow 0$ and the integral in (A4) are not interchangeable.

\section{APPENDIX B: A LIMIT THEOREM FOR AN INTEGRAL}

Let $f$ be a continuous function on $(0, \infty)$ such that for some $R>0$

$$
\int_{R}^{\infty}|f(x)| d x<\infty
$$

and the limit

$$
\lim _{x \rightarrow 0} x^{\lambda} f(x)=f_{0} \neq 0
$$

exists with some $\lambda \in \mathbb{R}$. Let $g$ be a bounded continuous function on $[0, \infty)$ such that

$$
\lim _{x \rightarrow 0} g(x) / x^{\mu} \neq 0
$$

exists with some constant $\mu>0$. For $t \in \mathbb{R}$, we consider the integral

$$
J(t)=\int_{0}^{\infty} f(x) g(t x) d x,
$$

which converges for

$$
\lambda<\mu+1 \text {. }
$$

The purpose of this appendix is to establish an elementary theorem concerning the asymptotic property of $J(t)$ as $t \rightarrow \infty$ :

Proposition B: (i) Let $\lambda<1$. Then

$$
\sup _{\kappa \in \mathbb{R}}|J(t)|<\infty \text {. }
$$

(ii) Let $\lambda=1$ and assume that $f, g \geqslant 0$ and

$$
\int_{0}^{\infty} \frac{g(x)}{x} d x=\infty
$$

Then

$$
\lim _{t \rightarrow \infty} J(t)=\infty .
$$

Moreover, for every $\epsilon \in(0,1)$, there exist positive constants $a$ and $b$ such that for all $t \in \mathbb{R}$

$$
J(t) \leqslant a t^{\epsilon}+b .
$$

In particular,

$$
\lim _{t \rightarrow \infty} J(t) / t^{\epsilon}=0 .
$$

(iii) Let $1<\lambda<\mu+1$. Then

$$
\lim _{t \rightarrow \infty} \frac{J(t)}{t^{\lambda-1}}=f_{0} \int_{0}^{\infty} \frac{g(x)}{x^{\lambda}} \text {. }
$$

Proof: Part (i) is obvious. (ii) Let $\lambda=1$. Then, by the change of variable $t x \rightarrow x$, we can write as

$$
J(t)=\int_{0}^{\infty} h_{1}\left(\frac{x}{t}\right) \frac{g(x)}{x} d x,
$$

where

$$
h_{\lambda}(x)=x^{\lambda} f(x) .
$$

By (B2) and Fatou's lemma, we have

$$
\lim _{t \rightarrow \infty} J(t) \geqslant f_{0} \int_{0}^{\infty} \frac{g(x)}{x} d x=\infty .
$$

Hence (B6) follows. To prove (B7), we write as

$$
J(t)=K_{1}(t)+K_{2}(t),
$$

where

$$
K_{1}(t)=\int_{0}^{R} f(x) g(t x) d x, \quad K_{2}(t)=\int_{R}^{\infty} f(x) g(t x) d x .
$$

Let

$$
C=\max _{x>0} g(x)>0
$$

and define

$$
\tilde{g}(x)=g(x) / C \text {. }
$$

Then we have $0 \leqslant \tilde{g}(x) \leqslant 1$, which implies that for $0<\epsilon<1$, $g(x) \leqslant C \tilde{g}(x)^{\epsilon}$. Using this inequality, we see that

$$
K_{1}(t) \leqslant C_{1} t^{\mu \epsilon},
$$

where

$$
C_{1}=C \sup _{x>0}\left(\frac{\tilde{g}(x)}{x^{\mu}}\right)^{\epsilon} \cdot \int_{0}^{R} x^{\mu \epsilon} f(x) d x<\infty .
$$

Obviously we have

$$
K_{2}(t) \leqslant C \int_{R}^{\infty} f(x) d x<\infty,
$$

which, together with (B11), gives (B7).

(iii) In this case also, we write $J(t)$ as (B10). By the change of variable $t x \rightarrow x$, we have

$$
\frac{K_{1}(t)}{t^{\lambda-1}}=\int_{0}^{\infty} \chi_{[0, t R]}(x) h_{\lambda}\left(\frac{x}{t}\right) \frac{g(x)}{x^{\lambda}} d x,
$$

where $\chi_{[0, t R]}$ is the characteristic function of the set $[0, t R]$. The integrand converges to

$$
f_{0}\left[g(x) / x^{\lambda}\right]
$$

for all $x>0$ as $t \rightarrow \infty$ and is dominated by

$$
\left(\sup _{0<y<R} \mid h_{\lambda}(y)\right) \frac{|g(x)|}{x^{\lambda}},
$$

which is integrable on $[0, \infty)$ with respect to $d x$. Thus we 
can apply the Lebesgue dominated convergence theorem to obtain

$$
\lim _{t \rightarrow \infty} \frac{K_{1}(t)}{t^{\lambda-1}}=f_{0} \int_{0}^{\infty} \frac{g(x)}{x^{\lambda}} d x .
$$

For $K_{2}(t)$, we have the estimate (B12) in the present case as well. Thus (B9) follows.

${ }^{1}$ W. Pauli and M. Fierz, Nuovo Cimento 15, 167 (1938).

${ }^{2}$ Foundation of Radiation Theory and Quantum Electrodynamics, edited by A. O. Barut (Plenum, New York, 1980).

${ }^{3}$ A. Arai, J. Phys. A: Math. Gen. 16, 49 (1983).

${ }^{4} \mathrm{H}$. Spohn, "Models of statistical mechanics in one dimension originating from quantum ground states," in Statistical Mechanics and Field Theory: Mathematical Aspects, Lecture Notes in Physics, edited by T. C. Dorlas et al. (Springer-Verlag, Berlin, 1986).

${ }^{5}$ H. Grabert, P. Schramm, and G.-L. Ingold, Phys. Rep. 168, 115 (1988).

${ }^{6} \mathrm{E}$. J. Moniz and D. H. Sharp, "Radiation reaction in nonrelativistic quantum electrodynamics," Phys. Rev. D 15, 2850 (1977). This paper discusses the Pauli-Fierz model without the dipole approximation both classically and quantum mechanically, concentrating its attention on the problem of runaway solutions and noncausal motion.

${ }^{7}$ A. Arai, J. Math. Phys. 24, 1896 (1983).
${ }^{8}$ D. P. L. Castrigiano and N. Kokiantonis, Phys. Rev. A 35, 4122 (1987).

${ }^{9}$ D. P. L. Castrigiano and N. Kokiantonis, J. Phys. A: Math. Gen. 20, 4237 (1987).

${ }^{10} \mathrm{~W}$. Eckhardt, Z. Phys. B-Condensed Matter 64, 515 (1986).

${ }^{\prime \prime}$ G. W. Ford, J. T. Lewis, and R. F. O'Connel, Phys. Rev. Lett. 55, 2273 (1985).

${ }^{12}$ G. W. Ford, J. T. Lewis, and R. F. O'Connel, Ann. Phys. (NY) 185, 270 (1988).

${ }^{13}$ N. G. van Kampen, Det Kongelige Danske Vid. Selskab Mat.-Fys. Medd. 26, (15), 1 (1951).

${ }^{14}$ A. Arai, J. Math. Phys. 22, 2539, 2549 (1981).

${ }^{15}$ A. Arai, J. Math. Anal. Appl. 140, 270 (1989).

${ }^{16}$ A. Arai, Hokkaido Math. J. 19, 1 (1990).

${ }^{17}$ E. B. Davies, Commun. Math. Phys. 33, 171 (1973).

${ }^{18}$ P. Schramm and H. Grabert, J. Stat. Phys. 49, 767 (1987).

${ }^{19}$ F. Schwabl and W. Thirring, Ergeb. Exakt. Naturwiss. 36, 219 (1964). ${ }^{20} \mathrm{~J}$. Glimm and A. Jaffe, Quantum Physics (Springer, New York, 1981).

${ }^{21}$ M. Reed and B. Simon, Methods of Modern Mathematical Physics II; Fourier Analysis, Self-adjointness (Academic, New York, 1975).

${ }^{22}$ A. Arai, J. Math. Phys. 30, 1277 (1989).

${ }^{23}$ E. C. Titchmarsh, Introduction to the Theory of Fourier Integrals (Oxford U.P., Oxford, 1948), 2nd ed.

${ }^{24} \mathrm{~F}$. Rocca, M. Sirugue, and D. Testard, Commun. Math. Phys. 19, 119 (1970).

${ }^{25} \mathrm{O}$. Bratteli and D. W. Robinson, Operator.Algebras and Quantum Statistical Mechanics I, II (Springer, New York, 1979, 1981). 
Journal of Mathematical Physics is copyrighted by the American Institute of Physics (AIP). Redistribution of journal material is subject to the AIP online journal license and/or AIP copyright. For more information, see http://ojps.aip.org/jmp/jmpcr.jsp Copyright of Journal of Mathematical Physics is the property of American Institute of Physics and its content may not be copied or emailed to multiple sites or posted to a listserv without the copyright holder's express written permission. However, users may print, download, or email articles for individual use. 